\title{
Information Feedback, Targeting, and Coordination: An Experimental Study
}

\author{
Matthew J. Hashim \\ mhashim@email.arizona.edu
}

\author{
Karthik N. Kannan \\ kkarthik@purdue.edu
}

\author{
Sandra Maximiano \\ maximiano@purdue.edu
}

April 4, 2016

\begin{abstract}
There are many contexts where an "everybody else is doing it" attitude is relevant. We evaluate the impact of this attitude in a multi-threshold public goods game. We use a lab experiment to study the role of providing information about contribution behavior to targeted subsets of individuals, and its effect on coordination. Treatments include one in which no information is provided and three others that vary in whom we provide information to: a random sample of subjects; those whose contributions are below the average of their group, and those whose contributions are above the average of their group. We find that the random provision of information is no different than not providing information at all. More importantly, average contributions improve with targeted treatments. Coordination waste is also lower with targeted treatments. The insights from this research are relevant more broadly to contexts including piracy, open innovation, and crowdfunding.
\end{abstract}




\section{Information Feedback, Targeting, and Coordination: An Experimental Study}

\section{Introduction}

Our research problem is originally motivated by the piracy context. Producers of digital products such as music, games, and software, argue that high piracy rates (e.g., an estimated $90 \%$ of games are pirated, 2D Boy, 2008; 95\% of music downloaded is pirated, IFPI, 2009) lead to huge losses (e.g., \$21.5B due to movie piracy). Yet, they also believe that pirates have an "everybody else is doing it, so maybe it is OK" attitude (Levin, 2011). These two actions are seemingly contradicting. On the one hand, information good producers invest in increasing the awareness about piracy with the hope of reducing it by mobilizing public opinion for regulatory or legal actions. On the other hand, the "everybody else is doing it' notion increases perceptions that 'piracy is all pervasive,' thus reducing the barriers to piracy. That is, consumers engage in piracy because they believe that it is being done by everybody else. Therefore, it not clear if sharing piracy information with consumers helps or harms producers of information goods. Effectively, our paper aims to answer the following research questions: does knowledge about others' extensive freeriding lead to more free-riding? Or, more importantly, can we shape overall free-riding behavior by skewing the provision of such information to only a certain subset of participants in the market? Even though our original motivation was the piracy context, the questions raised here also relate in an abstract fashion to other information systems domains where free-riding can potentially occur (including, for example, in the crowdfunding context, Zhang and Liu, 2012, and collaborations in open innovation projects, Zhang and Wang, 2012). In an abstract sense, we investigate how skewed provision of information about others' actions has outcome implications.

We address these questions from the perspective of the social welfare generated in the market, which is in turn dictated by individuals' actions. For example in the piracy context, arguments to limit piracy are made based on their social implications (e.g., the number of jobs lost, quality of the content produced, etc., Telang and Waldfogel, 2014). In crowdfunding contexts, arguably, platform owners such as Kickstarter are concerned about the welfare generated of the backers and creators. A setup where individual contributions have implications on the welfare generated can be studied using a coordination game. Specifically, we use a multi-threshold public good game in our case. ${ }^{1}$ One reason for choosing the public good game is that

\footnotetext{
${ }^{1} \mathrm{~A}$ (single) threshold public good game is one in which individuals need to coordinate to reach the provision point. Besides
} 
information goods-including music, games, and software-possess the characteristics of a public good (Varian, 1998). Even the crowdfunding context may be perceived to be similar to a public good context (Solomon et al., 2014). Furthermore, the multiplicity of thresholds in our game represent different quality levels that can be attained depending on how the individuals as a whole contribute to the public good. Such a characterization of quality levels is also consistent with claims in the piracy context that the quality of the digital good produced (e.g., features delivered, access to support resources) depends on the total of individual contributions (Cho and Ahn, 2010; Danaher et al., 2014; Jain, 2008). In such games, it turns out the actions that lead to social optima are also individually maximizing; but subjects are known to have problems coordinating to the optimal. Consequently, the aforementioned question becomes equivalent to: how does skewed information about free-riding in public good games affect the participants ability to attain the maximum social welfare?

Prior literature has shown that subjects in coordination games fail to coordinate effectively (i.e., do not attain the highest social welfare) and it has also considered mechanisms-such as the provision of information on other players' behavior - to improve coordination but with mixed results (see Section 2 for a more detailed literature review that also considers other mechanisms to improve coordination). Those studies that have analyzed information provision assume symmetric information. Even then it is not clear if subjects would react in a similar fashion to the provisioned information. For instance, if individuals have social preferences (e.g., altruism, reciprocity, efficiency) as experimental evidence seems to suggest, they may react differently to information. Therefore, provisioning information symmetrically may not improve coordination, and hence, the need to study the aforementioned question. It turns out that experimental research using a multi-threshold public good game is also scarce in general. Even in that setting, to the best of our knowledge, none have studied the impact of targeted information strategies.

For the analysis, we conduct a laboratory experiment to investigate how contributions and outcomes compare when information about others' contributions is targeted to subgroups of individuals as opposed to randomly providing information to subjects. We use the lab setting because it is difficult to obtain naturally occurring data of individuals' behavior under different information regimes. In some contexts even naturally occurring data are unreliable, for example, due to subjects lying about their behavior, or various

the equilibrium at no provision, all the possible combinations of contributions that assure the provision of the public good are also an equilibrium in a threshold public good game. By comparison, in a voluntary contribution mechanism (VCM), the group as a whole is best off when all members contribute to the public good. Each member, however, is better off by contributing nothing. Free-riding by all members is therefore the unique equilibrium in a continuous linear public good game. 
consequences arising from the Hawthorne effect (Landsberger, 1958). Furthermore, it is not easy to control the information flow as effectively in a real setting (or field experiment), as in an experimental lab. These problems are overcome through the use of a controlled setting and exogenous manipulation of information feedback in a laboratory environment. Randomization of our treatments allows us to tightly control possible confounding and selection effects that are typical with naturally occurring data.

We initially conduct our experiment by providing no feedback at all to the subjects so as to evaluate how similar the results from our multi-threshold game are with prior literature. We discover that, consistent with prior literature, subjects do find it difficult to coordinate. We follow up on our initial experiment by considering other information treatments, some of which were motivated by the "everybody else is doing it" attitude. Specifically, our analyses focus on the following three treatments: random feedback (i.e., contribution information is provided to a random sample of subjects), targeted above feedback (i.e., information is provided only to subjects whose contributions are above the average of their group), and targeted below feedback (i.e., information is provided only to subjects whose contributions are below the average of their group). We find that targeted information feedback results in the greatest level of contributions by subjects, whereas randomly providing information to subjects is ineffective. Between the two targeted treatments, sending information to those that are contributing on average more to the good, provides a higher level of contributions among the subjects. We also present insights about the group contribution information interacting with inequity aversion in creating the interesting outcomes.

The remainder of this paper is organized as follows. We review the literature in Section 2. Section 3 introduces our multi-threshold public good game and the equilibrium analysis of the game for our experimental parameters. Section 4 presents our information regimes, behavioral hypotheses, and experiment implementation details. Results are provided in Section 5, followed by a conclusion in Section 6.

\section{Related Literature}

Our paper relates to research that investigates the role of information about past contributions on cooperation and coordination in public goods games, ${ }^{2}$ and hence, we review the related literature in Section 2.1. In Section 2.2, we survey the related IS literature.

\footnotetext{
${ }^{2}$ An extensive experimental research on public goods provision has recognized the existence of free-riding behavior, heterogeneity in subjects' willingness to cooperate, and identified design features that affect cooperation in these games (see Davis and Holt, 1993, chap. 3, for an overview). A stream of experimental research on public goods has investigated mechanisms that promote cooperation and mitigate free-riding. For instance, decentralized exogenously imposed punishment and rewards (e.g., Fehr and Gächter, 2000), centralized experimenter imposed punishment and rewards (e.g., Croson et al., 2006), endogenously imposed institutions and group formation(e.g., Kosfeld et al., 2009), communication (e.g., Cason and Khan, 1999; Isaac and Walker, 1988).
} 


\subsection{Information Strategies for Cooperation and Coordination}

This subsection details the public goods experiments in the economics literature. Typically, the total group contribution level is revealed as feedback in such experiments. A few other variants have also been explored. In a voluntary contribution mechanism (VCM), Sell and Wilson (1991) compare the impact of providing no information, aggregate information, and individual level information about other members' contributions; and find that contributions are significantly higher with the individual level information than with the other two, which are not statistically different between them. Weimann (1994) finds results inconsistent with Sell and Wilson (1991). He finds no significant difference in the mean contributions when subjects were provided with the individual level information compared to the average contribution of their group (i.e., aggregate information). To reconcile the two results, Croson (2001) also considers a VCM with feedback at the aggregate and individual levels. Even though they find no significant differences in the average contributions between the two levels, the individual level feedback generated a higher variance in the contributions.

Variants of the individual and the aggregate level contributions feedback have been analyzed separately. For example, Croson and Marks (1998) find that anonymity decreases contribution amounts and increases the variance. Also, when information on individual contributions is probabilistically revealed, Anderson and Stafford (2009) find that the a priori possibility of an announcement increases public contributions slightly, whereas the ex post possibility only appears to promote free-riding. The individual level information feedback has also been studied in other coordination games such as the minimum-effort games ${ }^{3}$ but the results are mixed even there. Berninghaus and Erhart (2001) and Brandts and Cooper (2006) show a positive effect on coordination, while Devetag (2005) and Van Huyck et al. (1990) find that ex post information does not solve mis-coordination problems.

In a field study of on-air fund-raising for a public radio station, Shang and Croson (2009) compared different variants of revealing aggregate information. Providing the $90^{\text {th }}$ and $95^{\text {th }}$ percentile information to potential donors increased the level of overall contributions and also was effective with first-time donors. Similarly, a natural field experiment in the context of public utilities was conducted by Ayres et al. (2012), who shows that information about energy usage presented in 'peer comparison reports' is effective at modifying the behavior of high energy use consumers, significantly reducing their consumption.

\footnotetext{
${ }^{3}$ In minimum-effort games, group performance depends on the behavior of the 'weakest link'. A dilemma arises when the player has to decide between a safe choice of minimal effort which maximizes the minimum possible payoff and the choice of maximal effort which maximizes the total group earnings. See Devetag and Ortmann (2007) for an overview on coordination failure.
} 
Our paper investigates the effect of information about previous contributions on coordination just like many other prior works, but ours does so in a multi-threshold public goods context (we provide in Section 3 more details regarding why the multi-threshold public good game is appropriate for our context). Experimental research using a multi-threshold public good game is scarce in general. To the best of our knowledge, only sequentially attained thresholds ${ }^{4}$ have been studied by the prior literature in this context (Bagnoli et al., 1992; Normann and Rau, 2011). We are unaware of any study on the impact of targeted information on coordination in a public good game (including a multi-threshold one).

\subsection{Related Information Systems Literature}

The IS literature is increasingly using experimental economics in various contexts, such as auctions (e.g., Adomavicius et al., 2012, 2013; Bapna et al., 2010; Cason et al., 2011), online dating (e.g., Bapna et al., 2012), and privacy (e.g., Brandimarte et al., 2010; Tsai et al., 2011). As regards the digital piracy context, we are only aware of Hashim et al. (2014), where the role of the source of advice on piracy decisions is investigated. In the following paragraphs we provide an overview of the economics of digital piracy as well as the crowdfunding literatures.

Most economics of piracy papers demonstrate the importance of pricing digital goods and the associated effects on piracy decisions (Chen and Png, 2003; Khouja and Park, 2008). Accordingly, pricing decisions are often studied in conjunction with other factors. For example, Sundararajan (2004) focus their attention on the use of technology deterrence in preventing piracy. Product bundling has also been explored in limiting piracy when used in conjunction with deterrence strategies (Bhattacharjee et al., 2009; Gopal and Gupta, 2010). Chellappa and Shivendu (2005) capture the desire for pirates to sample digital goods, and model that aspect along with pricing considerations. Liu et al. (2011) has taken into account word-of-mouth effects with optimal software pricing. Impacts to the level of quality of information goods developed by vendors has also been modeled in the presence of piracy (Lahiri and Dey, 2013). Patch management has also been investigated to consider the impacts of restricting or allowing pirated software users to receive security patches (August and Tunca, 2008; Kannan et al., 2013; Lahiri, 2012). By doing so, they provide policy guidance for vendors as well as social planners under various piracy contexts. As alluded to earlier, antipiracy and information feedback mechanisms do not appear to have been studied before.

\footnotetext{
${ }^{4}$ The subjects are presented with a first decision to meet a threshold of contributions required for the provision of the first unit of the public good. If the threshold is met, the subjects then must decide to pursue the second threshold of the public good, and so forth.
} 
As already mentioned, the problem studied here can also relate to the crowdfunding context. There have been prior works that have established that the crowdfunding context relates a public good game (Solomon et al., 2014; Wash and Solomon, 2014). A number of papers have considered the factors that affect contributions in the public good game. In that regard, Burtch et al. (2014) use a field experiment to how the lack of anonymity for the contributors affects contribution behavior. Herzenstein et al. (2011); Zhang and Liu (2012) show that 'rational' herding can occur. On the other hand, Burtch et al. (2013) show that crowding out effects can also occur when contributions are less important to the recipient of the funds. Lin et al. (2013) study how information about borrowers' friends serves as a signal of credit quality that affects contributions. The analysis in our paper relates to how such contributions may be affected because of a subset of the segment knowing about others' contributions.

Free-riding on the efforts of others is also a concern in the open innovation context (Johnson, 2002). Fang and Neufeld (2009) show that collaborator effort is sustained if collaborators strive to improve both the welfare of the community and the software code. Other motivational factors have also been considered. Roberts et al. (2006) show that intrinsic and extrinsic rewards improve the quality of contributions to software projects. Also, collaborator ties sustain open source projects when the ties are based upon pastperformance on projects (Hahn et al., 2008; Zhang and Wang, 2012). Our paper relates to these works because using targeted information as a mechanism to sustain collaborator effort does not appear to have been studied before in open innovation. In the following section, we describe the basic multi-threshold public good game we use for the experiment.

\section{The Multi-Threshold Public Good Game: Theory and Experimental Results}

The purpose of this section is three-folded. We present in Section 3.1 the single period multi-threshold public good game and the parameters we use in the experiment. Since the game we propose is also a contribution to the body of knowledge, for the sake of completeness, we provide the theoretical predictions in Section 3.2. Lastly, we evaluate in Section 3.4 using an experiment described in Section 3.3 how the attainment of equilibrium is in this new game when the information provision is similar to the prior literature. In other words, we ensure that changing the details of the game does not change the subjects' ability to attain the equilibrium. We believe that is important to check this aspect before studying the impact of targeted information. The results show that players are not able to reach or maintain equilibrium outcomes in the multi-threshold public good game. The main takeaway from the analysis is that coordination is quite difficult 
in the absence of any kind of mechanism that improves the ability for players to coordinate efficiently.

Before we describe the game, we mention why a multi-threshold game is best suited to our context. First, as opposed to a single-threshold game, the multiplicity of thresholds allows for a larger possibility of equilibria-from inefficient to efficient ones-allowing for a more precise comparison of the impact of different information strategies. Second, between the single- and multi-threshold games, ordinal comparisons of policies may even be reversed. ${ }^{5}$ Third, compared to the continuous public good games, multi-threshold games have an advantage in that the Pareto-optimal outcomes are supportable as Nash equilibria (Palfrey and Rosenthal, 1984; Bagnoli et al., 1992). Next, we describe the game.

\subsection{The Multi-Threshold Public Good Game}

We consider $n$ symmetric individuals concerned with the provision of a single unit of a public good with a specific quality. The quality can be one of the elements of the ordered set $\mathcal{Q}$ and is represented by an index variable $q$ (starting at 1). Each consumer is endowed with an initial amount $E_{i}$, which can either be invested in a private account or used towards the provision of the public good. Every individual, $i$, simultaneously and independently chooses to contribute $x_{i}$ from their initial endowment to the public good.

The individual earnings from investing in the private account are simply $E_{i}-x_{i}$. The individual earnings from consumption of the public good depend on the quality of the public good. A public good of quality $q$ is provided in a threshold setting with a cost of $\underline{\mathrm{X}}^{q}$. So, only if $\sum_{i} x_{i} \geq \underline{\mathrm{X}}^{q}$ units, the quality provided is $q$. The costs are increasing in quality, i.e., $\underline{X}^{q+1}-\underline{X}^{q}>0$ and because $\underline{X}^{1}=0$, a minimum quality is always assured for the public good. The payoff function for individual $i$ is linear and given by:

$$
\pi_{i}\left(E_{i}-x_{i} ; v_{i}^{q}\right)=E_{i}-x_{i}+v_{i}^{q} \quad \text { if } \quad \underline{X}^{q} \leq \sum_{i=1}^{n} x_{i}<\underline{X}^{q+1}
$$

where $v_{i}^{q}$ refers to individual $i$ 's value for a single unit of a public good of quality $q$. Payoff derived increases in $q$, with $v_{i}^{q+1}>v_{i}^{q} \geq 0$. Furthermore, the earnings from the public good component are symmetric, i.e., $v_{i}^{q}=v_{j}^{q}$ for $j \neq i .^{6}$ So, that earning is simply referred to as $v^{q}$.

\footnotetext{
${ }^{5}$ For example, suppose that in a single threshold public good setting, the public good is provided both under information strategies A and B, but the provision is more wasteful in the strategy B as individuals contribute much more above the threshold as compared to information strategy A. In this example, information strategy B seems more preferable than A. This conclusion may be reversed in a multi-threshold setting if under information strategy B individuals would be able to reach a higher possible provision point.

${ }^{6}$ Individuals are assumed to only care about their private account and the quality level of the public good. They are assumed not to have warm glow feelings from contributing to the public good. Therefore, the individual's payoff does not depend directly on a contribution the individual makes to the public good (Andreoni, 1990).
} 
Our setting does not contemplate refunds or rebates. More specifically, contributions are not returned to their contributors when the provision point is not met. Also, contributions are not returned when they exceed a certain threshold but are insufficient for the next quality level. ${ }^{7}$

In the following subsection, we analyze the equilibrium of this game. The equilibrium analysis discussions are kept generic but whenever needed, we use the parameters from our experimental setup as an example. In our experiment, we consider $n=5$ players, each with an initial endowment of $E_{i}=50$. Table 1 shows the earnings from the public good and the various thresholds. Our choice of parameters is such that

Table 1: Experimental Parameters

\begin{tabular}{|c|c|c|c|c|c|c|}
\hline Range & $q$ & $\begin{array}{c}\text { Quality } \\
\text { Descriptions }\end{array}$ & $\begin{array}{c}\underline{X}^{q}(\% \text { of } \\
\text { endowment })\end{array}$ & $\begin{array}{c}\text { Group } \\
\text { payoff }\left(v_{i}^{q}\right) \\
\text { for the good }\end{array}$ & $\begin{array}{c}\text { Step return } \\
\frac{n v^{q}}{X^{q}}\end{array}$ & $\begin{array}{c}\% \text { of } \\
\text { threshold to } \\
\text { be willing to } \\
\text { contribute }\end{array}$ \\
\hline $0 \leq \sum_{i} x_{i}<50$ & 1 & Poor & $0(0 \%)$ & $0(0)$ & Indeterminate & $0 \%$ \\
\hline $50 \leq \bar{\sum}_{i} x_{i}<100$ & 2 & Medium & $50(20 \%)$ & $92.5(18.5)$ & 1.850 & $63.0 \%$ \\
\hline $100 \leq \sum_{i} x_{i}<150$ & 3 & Good & $100(40 \%)$ & $227.5(45.5)$ & 2.275 & $73.0 \%$ \\
\hline $150 \leq \sum_{i} x_{i}<200$ & 4 & Very Good & $150(60 \%)$ & $405(81)$ & 2.700 & $76.3 \%$ \\
\hline $200 \leq \sum_{i} x_{i}$ & 5 & Excellent & $200(80 \%)$ & $625(125)$ & 3.125 & $78.0 \%$ \\
\hline
\end{tabular}

the step return (SR), which is the ratio of the aggregate group value from consuming a certain quality public good to their share of the cost, $S R=\frac{n v^{q}}{\underline{X}^{q}}$ is greater than 1. Otherwise, it would not be worth producing that quality level. Note also that the step returns are higher when the quality of the public good is higher. Individuals therefore have an incentive to coordinate to a higher quality level.

\subsection{Theoretical Predictions}

In this subsection we discuss the theoretical predictions under two assumptions about social preferences: 1) individuals care only about their own earnings, and 2) individuals also care about the earnings of others. In particular, they care about how their own monetary payoff compares with the monetary payoffs of others.

First, consider narrow self-interest preferences. In equilibrium, the individual decision on how much to contribute to the public good depends on how much her contribution is crucial for the provision of a certain quality level. Denote by $X_{-i}$ the sum of the individuals' contributions of everyone except $i$. Contribution of zero is the best response of individual $i$ whenever $\underline{\mathrm{X}}^{q-1} \leq X_{-i} \leq \underline{\mathrm{X}}^{q}-E_{i}$, for all $q$ and $q-1$ that are indexes of $\mathcal{Q}$. Individual $i$ 's contribution is crucial for provisioning a public good of quality $q$ if and only

\footnotetext{
${ }^{7}$ Given that our focus is to investigate the impact of information strategies on coordination we needed a setting in which coordination is particularly difficult. Refunds and rebates, however, have been shown to improve coordination and as such we did not contemplate them (Isaac et al., 1989).
} 
if $\underline{\mathrm{X}}^{q}-E_{i} \leq X_{-i} \leq \underline{\mathrm{X}}^{q}$. Being crucial is necessary but not sufficient for $i$ 's contribution. No individual contributes any positive amount to the public good if her individual gain from extra quality is not positive, i.e., the individual rationality constraint, $x_{i} \leq v_{i}^{q}-v_{i}^{q-1}$ has to hold. It follows that the decision rule for each individual $i$ is:

$$
x_{i}=\left\{\begin{array}{l}
\underline{\mathrm{X}}^{q}-X_{-i} \quad \text { if } \quad \underline{\mathrm{X}}^{q}-E_{i} \leq X_{-i} \leq \underline{\mathrm{X}}^{q} \quad \text { and } \quad X_{-i} \geq \underline{\mathrm{X}}^{q}-\left(v_{i}^{q}-v_{i}^{q-1}\right) \\
0 \text { otherwise }
\end{array}\right.
$$

Assuming that a public good with quality $q$ can be provided, i.e., the group has enough endowment so as to make an equilibrium level feasible and the value to the entire group of the provision of such quality is larger than the cost, i.e, $\sum_{i=1}^{n} v_{i}^{q} \geq \underline{\mathrm{X}}^{q}$, there is a continuum of pure Nash equilibria ${ }^{8}$ consisting of all feasible situations where quality thresholds are met. In particular, these equilibria consist of all vectors of $x_{i}$ satisfying the efficiency constraint: $\sum x_{i}=\underline{\mathrm{X}}^{q}$ and the individual rationality constraint: $x_{i} \leq$ $v_{i}^{q}-v_{i}^{q-1}$. These equilibria can be symmetric or asymmetric depending on the cost-sharing rule. In a symmetric equilibrium $x_{i}=x_{j}, \forall i, j$, while in an asymmetric equilibrium, at least $\exists i$ and $j$ s.t. $x_{i} \neq x_{j}$. Note that the symmetric equilibria in our game are not necessarily payoff equivalent. Also, the Paretoefficient equilibria maximizes the step return.

Corresponding to the parameters of our experimental setup, the set of pure Nash equilibria are as follows: 1) $x_{i}^{*}=0$ and $q^{*}=$ poor; 2) $x_{i}^{*}=[0,18.5], \sum_{j=1}^{n} x_{i}=\underline{\mathrm{X}}^{2}$, and $q^{*}=$ medium; 3) $x_{i}^{*}=[0,27], \sum_{j=1}^{n} x_{i}=$ $\underline{\mathrm{X}}^{3}$, and $q^{*}=\operatorname{good}$; 4) $x_{i}^{*}=[0,35.5], \sum_{j=1}^{n} x_{i}=\underline{\mathrm{X}}^{4}$, and $\left.q^{*}=\operatorname{very} \operatorname{good} ; 5\right) x_{i}^{*}=[0,44], \sum_{j=1}^{n} x_{i}=\underline{\mathrm{X}}^{5}$, and $q^{*}=$ excellent.

Next, assume that agents are motivated by inequity aversion as in Bolton and Ockenfels (2000). In particular, individuals not only care about their own monetary payoffs but also how their payoffs compare with the average of those of other subjects. ${ }^{9}$ For an $n$-player game with monetary payoffs $y=\left(y_{0}, \ldots, y_{n}\right)$, player $i$ maximizes the expected value of her motivation function, $\varphi_{i}=\varphi_{i}\left(y_{i}, \sigma_{i}\right)$ where $\sigma_{i}$ is $i$ 's relative share of the payoffs:

$$
\sigma_{i}\left(y_{i}, \sum_{j=1}^{n} y_{j}, n\right)= \begin{cases}\frac{y_{i}}{\sum_{j=1}^{n} y_{j}} & \text { if } \sum_{j=1}^{n} y_{j}>0 \\ \frac{1}{n} & \text { if } \sum_{j=1}^{n} y_{j}=0\end{cases}
$$

\footnotetext{
${ }^{8}$ Both pure and mixed equilibria are feasible but we focus on pure Nash equilibria only.

${ }^{9}$ We consider this model because in our experiment subjects do not know other players' individual contributions and in case they get information that will pertain the group average.
} 
The motivation function is assumed to be continuous and twice differentiable, concave in the relative argument with a maximum achieved when player $i$ 's own share is equal to the average share (social reference point). In particular, consider the additively separable motivation functional form for a $n$-player game (cf. the 2-player example in Bolton and Ockenfels, 2000, page, 173):

$$
\varphi_{i}\left(y_{i}, \sigma_{i}\right)=a y_{i}-\frac{b}{2}\left(\sigma_{i}-\frac{1}{n}\right)^{2}
$$

where $a \geq 0$ and $b>0$. In this expression the parameter $a$ measures the extent to which a player cares about his own monetary payoff. Parameter $b$ reflects the concern for earning more or less than the average earnings of the group. The further $y_{i}$ is from the average, the higher the loss from the relative term. This function nests to two extreme cases: 1) If $a=0$, the player exhibits strict relativism and achieves maximum utility at the social reference point; 2) If $b \rightarrow 0$, the player has narrow self-interest preferences.

The value of $\frac{a}{b}$ is thus decisive for player $i$ 's contribution to the public good in response to the total contribution of others. Whenever $b \neq 0$, the set of all possible Nash equilibria is smaller as compared to the set of equilibria when players exhibit narrow self-interest. ${ }^{10}$ Figure 1 illustrates these predictions for our experimental parameters. The solid black lines refer to the standard Nash equilibria where the combined allocation of tokens for the group account exactly satisfies a given threshold. The solid gray lines exemplify the existence of inequity aversion preferences, where the $\frac{a}{b}$ determines the number of asymmetric equilibria. If all players show a preference for strict relativism, i.e., $a=0$, there are only symmetric equilibria (the crosses along the $45^{\circ}$ line). The gray line can move downwards or upwards in case players do not suffer equally from advantageous and disadvantageous inequity (if players are motivated by inequity aversion as in Fehr and Schmidt, 1999).

The above discussion serves to show that the equilibrium outcomes can vary depending on whether individuals have pro-social concerns. In particular, if they have inequity aversion preferences, the set of equilibrium outcomes may be reduced. A similar picture would be obtained if individuals are motivated by conditional cooperation (Falk et al., 2000) and cooperate as long as others do. Also, if individuals have efficiency concerns (see e.g., Engelmann and Strobel, 2004), the set of equilibria may shrink as there is a

\footnotetext{
${ }^{10}$ This prediction holds in the case where players are homogeneous with respect to $\frac{a}{b}$, as well as when there is heterogeneity in the population with respect to $\frac{a}{b}$. There $\exists \frac{a}{b}$ for $\forall n$ that gives the same Nash solution as in the case where for players have a different $\frac{a}{b}$.
} 


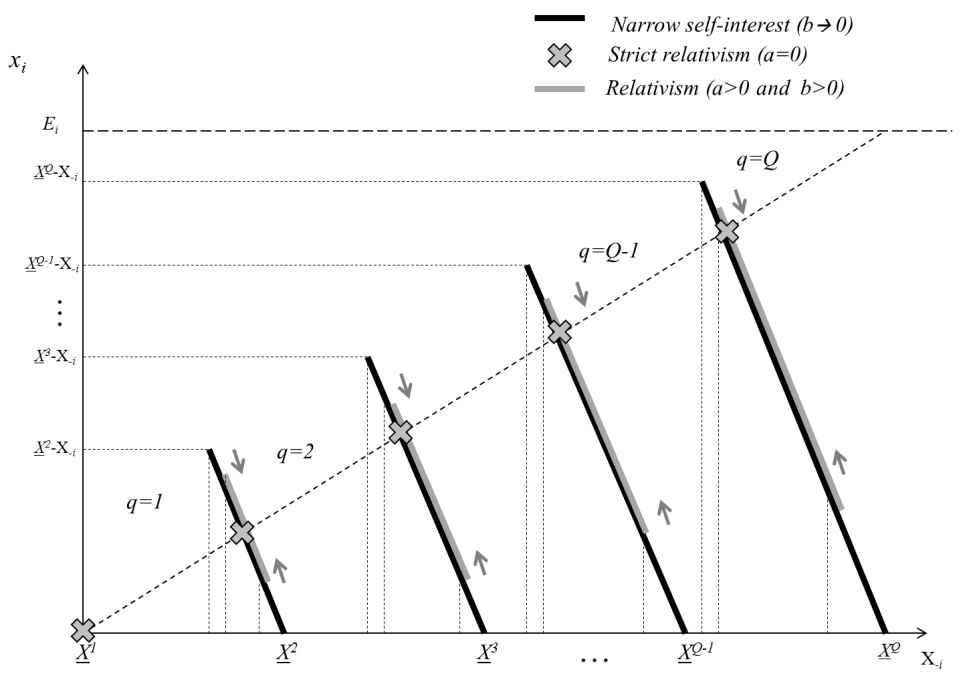

Figure 1: Set of Nash Equilibria for Self-Interest Preferences and Inequity Aversion, where $Q=|\mathcal{Q}|$

tendency to move towards the most efficient outcome. For instance, if individuals' concern for efficiency is very large we may end up with equilibria only at excellent quality, i.e., at the highest line in Figure 1. Our experiment, however, is not designed to test different models of social preferences. A large number of studies have already established the importance and the limitations of other motivational factors behind subjects' choices and have designed experiments to discriminate between different models (e.g., Charness, 2004; Cox, 2004; Falk et al., 2000; McCabe et al., 2003).

\subsection{Experimental Implementation of the Multi-Threshold Public Good Game}

In the experiment we implemented the multi-threshold game as described in Section 3.1. As mentioned earlier, we set $n=5, E_{i}=50$, and used the threshold and quality parameters as in Table $1 .{ }^{11}$ Subjects play the game repeatedly for 16 rounds under a strangers matching protocol. Therefore, at the beginning of each round, subjects are randomly re-matched with a different group of subjects. Subjects are never informed of who is in their group or about individual contributions of their group members. There are various reasons for choosing a strangers matching. First, it enables us to most appropriately capture the equilibrium for the one-shot game (Andreoni and Croson, 2008; Croson, 1996). Second, it avoids reputation effects and therefore makes coordination harder for the subjects. It is well known that the partner design itself enables higher coordination in a public good context. Finally, many real settings consider individuals that do not

\footnotetext{
${ }^{11}$ Prior literature has shown that the findings from the public goods experiments are consistent for various group sizes, including very large groups (Isaac et al., 1994). For the findings to hold, it is important to isolate the causal effect of interest in a lab experiment, and that causal effect should not change across group sizes (Falk and Heckman, 2009).
} 
necessarily interact with the same groups of people.

At the beginning of each round, each subject receives an endowment. ${ }^{12}$ Next, subjects are asked about their beliefs regarding the average contribution in their group. Belief elicitation was not incentivized in order to avoid any interference of the elicitation process with contribution behavior (Gächter and Renner, 2010).

Subsequently, all subjects are reminded of their own allocation rate from the prior round. Subjects then choose their individual contribution to the public good, $x_{i}$. For $x_{i}$, subjects were allowed to only enter integers in $[0,50] .{ }^{13}$ Once all of the subjects input their contributions, the quality level attained and the individual profit for the round are displayed to each subject. Recall, however, that subjects are never informed about their group members' individual contributions to the public good. The aforementioned procedures are repeated for every round. ${ }^{14}$

The experiment was conducted at the Vernon Smith Experimental Economics Laboratory (VSEEL) at Purdue University in the spring of 2010. Subjects were recruited by email using the laboratory's on-line recruitment system, and subject participation was limited to a single session. The computerized experimental environment was implemented using z-Tree (Fischbacher, 2007). A total of 20 subjects participated in the No Info treatment, and they were randomly assigned to individual computers. Communication was not allowed during the session. Copies of the experiment instructions were provided to each subject and were read aloud by the experiment administrator. A copy of the instructions used to conduct the experiment is available in Appendix A. Completion of control questions was required to ensure each subject understood the experimental procedures prior to starting the actual experiment. Any subject that failed to answer the control questions after three attempts was personally assisted by the experiment administrator.

\subsection{Results}

In this section we present the results for rounds 1-16 of our No Info treatment, which implements the game as described in Section 3.3. In total we consider 320 observations. The average contribution and the average quality was 24.72 and 2.94 respectively. Figure 2(a) shows the average contribution per round and Figure 2(b) the average quality per round. The aggregate picture hides group behavior and also fails to account

\footnotetext{
${ }^{12}$ The purpose for providing the endowment each round is to avoid exposure to potential risk due to the subjects' prior performance, as well as maintain the non-repeated design in our game.

${ }^{13}$ Input was validated by the computer and subjects were shown a warning message if they attempted to violate the interval provided. Validation also includes entering a non-integer value.

${ }^{14}$ The implementation of our game as described consists of our No Info treatment. We conducted an additional three treatments that are described in Section 4, in which subjects may receive extra information. The implementation of those treatments is presented in Section 4.3.
} 
for group heterogeneity. In 64 groups of subjects, 1 group obtains poor quality, 16 medium, 34 good quality, 12 very good, and 1 excellent. In the No Info treatment the quality alternates on average between Medium and Good across rounds.

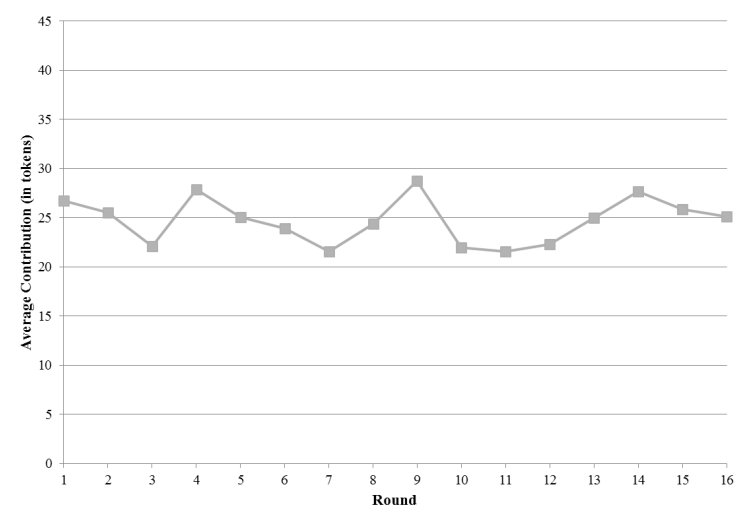

(a) Average Contribution per Round

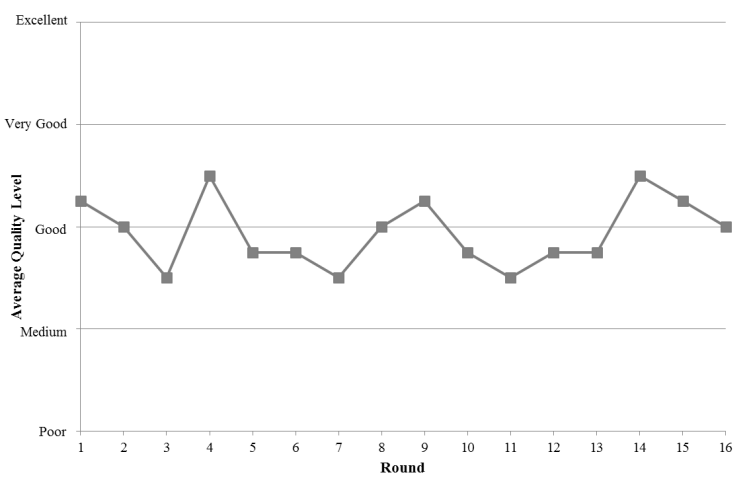

(b) Average Quality Level per Round

Figure 2: Per Round Measures for No Info Treatment

Next we look at the efficiency and waste generated. We define efficiency as the surplus achieved relative to the maximum feasible surplus:

$$
\eta=\frac{n v_{i}^{q}-\sum_{i=1}^{n} x_{i}}{n v_{i}^{|\mathcal{Q}|}-\underline{X}^{|\mathcal{Q}|}}
$$

and waste as the excess of the contribution above the threshold needed for the quality level achieved:

$$
w=\sum_{i=1}^{n} x_{i}-\underline{X}^{q}
$$

Recall that our multi-threshold game has increasing step returns on the thresholds. The efficiency $(\eta)$ is less than $100 \%$ whenever the combined contributions fail to produce the highest quality public good. If subjects can coordinate to a particular level without any waste (i.e., $w=0$ ), then the efficiency level corresponding to our experimental parameters for Poor quality is zero, for Medium quality is $10 \%$, for Good quality is $30 \%$, and $60 \%$ for a Very Good quality level. Excess $w$ further decreases $\eta$.

Table 2: Average Waste and Count of Groups Coordinating at NE in Rounds 1-16

\begin{tabular}{lcccccc}
\hline & Poor & Medium & Good & Very good & Excellent & Total \\
\hline Average Waste & 32.00 & 34.13 & 26.91 & 17.75 & 3.00 & 26.70 \\
Groups at NE & 0 of 1 & 1 of 16 & 0 of 34 & 0 of 12 & 0 of 1 & 1 of 64 \\
\hline
\end{tabular}

The average efficiency is 0.25 with an average waste equal to 26.70 tokens, indicating that subjects fail 


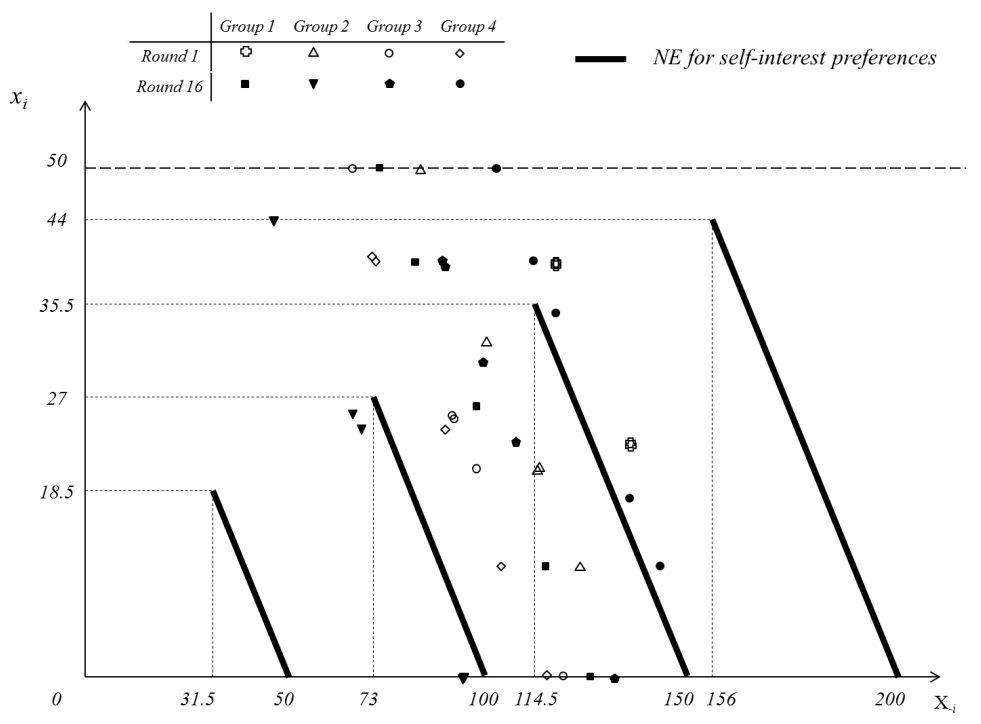

Figure 3: Plot of $x_{i}$ and $X_{-i}$ for each Subject (Round $1 \&$ Round 16)

to coordinate at equilibrium levels. As an illustration of the difficulty of coordinating contributions, Figure 3 plots contributions $\left(x_{i}\right.$ by $X_{-i}$ ) for Round 1 and for Round $16 .{ }^{15}$ Consistent with Figure 1, the solid black lines refer to the standard Nash equilibria where the combined allocation of tokens for the group account exactly satisfies a given threshold. It is clear from the figure that many subjects contribute substantially greater tokens than other players in their group, as shown by the markers plotted $\geq 40$ tokens. Also, each marker is either well above or below any given threshold. Further, Table 2 provides the average waste and count of groups coordinating at a NE for Rounds 1-16. It is clear from the table that nearly all groups (with the exception of 1 group observation) are unable coordinate at a NE without waste, and the waste accordingly varies from 3 to 34.13 tokens on average per quality level. Given that coordination is quite difficult in the multi-threshold public good game without any kind of information provided to the subjects about their group members, we next introduce information feedback and associated behavioral hypotheses.

\section{Information Regimes and Behavioral Hypotheses}

The equilibrium solution presented in Figure 1 is independent of whether information about the average outcome of other players is provided and how it is provided. This holds because we assume that individuals are rational and aim at maximizing their payoff function (independently of their preferences), and can form correct beliefs about their group members' contributions. Results presented previously, however, highlight

\footnotetext{
${ }^{15}$ Unfilled markers represent contributions in Round 1 and solidly-filled markers represent contributions in Round 16. Each marker plots a subject's contribution $\left(x_{i}\right.$, vertical axis) by the sum of the other group members' contributions $\left(X_{-i}\right.$, horizontal axis).
} 
how difficult it is for subjects to coordinate in a simultaneous multi-threshold public good game. Results show that subjects almost never play in the equilibrium, there is significant waste of resources, and outcomes are far from being efficient.

Under this scenario, information feedback about group members' contributions may be used as a coordination device. In fact, information feedback has been shown to aid coordination, even in a repeated game with random-matching. For example, in a repeated random-matching prisoner's dilemma game, information on the current partners' past actions can theoretically be enough to sustain any level of cooperation (Takahashi, 2010). Moreover, if information feedback may be used as a coordination device, to whom information is provided can make a difference. Therefore, in this section we present and motivate the information regimes we implement and discuss the related behavioral hypotheses. Given our previous results and our main goal of studying the implications of targeted information feedback on coordination and cooperation, to derive our behavioral hypotheses we do not assume any equilibrium play.

Based on the anecdotal evidence regarding the "everybody else is doing it" attitude, it appears that targeting of information should play a role. In this section we develop the related hypotheses.

\subsection{Information Regimes (Treatments)}

Our study involves four information regimes. First, as discussed in the previous section, we conduct a No Info regime, in which no information feedback is provided across the rounds. In each of the other three regimes, before individuals make their contribution decisions, a subset of subjects in each group is informed about the average previous round contribution of their actual group members. ${ }^{16}$ The subset that receives information differs across the three regimes. In the Random Info regime, the subset of subjects to receive the information is randomly decided. In the Target Below (Target Above) regime, the subjects to receive the information are those whose previous round contributions are below (above) the average previous period contribution of their present group members.

Despite that the information about others' contributions may help subjects to coordinate, it is not clear if the information would affect each individual in the same way. More specifically, if individuals have social preferences, such as inequity aversion, and/or are conditional cooperators, this information is likely to make their preferences more salient and, indeed, to whom information is provided may have an effect on individuals' choices. These preferences motivate our Target Below and Target Above treatments. As our

\footnotetext{
${ }^{16}$ Recall that players are randomly matched with different partners every round. The design details will be further discussed in Section 4.3 .
} 
main goal is to investigate the effect of targeting information, the best control treatment would not be the one in which we do not provide information, but instead a treatment in which subjects would still receive information but not in a targeted manner (i.e., Random Info). Moreover, we need to keep the same amount of information in the the non-targeted treatments in order to make comparisons. Both of these concerns justify our choice for conducting a treatment in which information is randomly provided to a subset of subjects.

\subsection{Behavioral Hypotheses}

As previously shown, subjects are not playing according to equilibrium predictions. The mis-coordination is likely to be due to incorrect beliefs about their group's contribution behavior and how crucial their own contribution is in achieving a certain quality level. Therefore, when information about the group's contribution behavior is provided, subjects may revise their estimate about how crucial their contribution is, and change their individual contribution level. In our game, if subjects receive information and use it to correctly revise their estimates about others' contributions, we expect them to coordinate more efficiently, i.e., with less waste, and possibly, to a higher quality level. ${ }^{17}$

Hypothesis 1. Treatments where information is provided achieve higher efficiency than No Info.

The effect of targeting strategies on coordination depends on the type of preferences we assume the subjects to have. First, consider that players care only about their monetary payoffs. In this case, even if information feedback aids in coordination, we do not expect differences in coordination across the information treatments (i.e., Random Info, Target Below, Target Above). That is, targeting information to those either below or above the average contribution of the group should make no difference in the individuals' ability to coordinate to an equilibrium point, as compared to what may happen in Random Info. We hypothesize so because if subjects do not have social preferences they would not put any particular weight on having contributed more or less than the average. Also, we have no reason to a priori expect any difference either in the number of subjects that contributed below or above the average, or in the size of the deviations of individual contributions to the group average across treatments.

Individuals, however, may have social preferences, such as an aversion for inequity, a preference for conformity (i.e., "everybody else is doing it" attitude), or exhibit reciprocal behavior. In this case, information about the average contribution of their group members may make inequality concerns more salient and,

\footnotetext{
${ }^{17}$ Note, that although it makes sense that information may help subjects to coordinate with less waste, it is not clear whether a higher quality level can be achieved, as all possible quality levels can be sustained in the equilibrium.
} 
therefore, targeting information may influence subjects' decisions differently across treatments. In particular, consider that individuals have a preference for strict relativism (cf. Bolton and Ockenfels, 2000) and dislike earning more or less than the average (see, Section 3.2), we expect the targeted treatments to result in a different level of coordination compared to Random Info. If a player observes a low average contribution level in Random Info in comparison with what she expects to contribute, her optimal choice-given her inequity-aversion preferences - consists of decreasing her own contribution. However, if a player observes a high average contribution level in Random Info in comparison with what she expects to contribute, the player may encounter disutility from other players doing worse than oneself, resulting in an increase in her own contribution. Because these adjustments will be simultaneously made, we expect no differences in the quality provided in the Random Info treatment as compared to the quality in the No Info treatment. We do, however, expect a convergence to a more symmetric contribution level. ${ }^{18}$

Hypothesis 2. If individuals have strong inequity averse preferences or have strong reciprocal concerns, we expect more symmetric contributions (but not necessarily a higher quality) in Random Info than in No Info.

For the same reasons mentioned above, we believe the targeted feedback treatments will also reach more efficient equilibria. However, we expect differences in the average contributions. In Target Below, the subjects contributing below the average will first move along the solid black threshold lines shown in Figure 1 to a more symmetric rate of contribution. Because subjects above the average do not receive any information feedback, their behavior is not expected to change. Consequently, in Target Below, it is possible to observe a higher quality level along time, and also more asymmetric contributions. The opposite result is expected for the Target Above treatment.

Hypothesis 3. If individuals have strong inequity averse preferences or have strong reciprocal concerns, we expect progressively higher contributions (quality level) in the Target Below treatment and lower contributions (quality level) in the Target Above treatment.

An implication of Hypothesis 3 is that the average contribution is higher with Target Below than with Target Above.

\footnotetext{
${ }^{18}$ The same prediction holds if subjects have reciprocal preferences. More specifically, a conditional cooperator derives utility from contributions of others and in particular, from rewarding high contributions with a higher contribution and punishing lower contributions of others with a lower contribution. A similar explanation holds if subjects have conformity preferences as well.
} 
Our behavioral hypotheses assume that subjects who do not receive information do not make any particular adjustments to their behavior. However, given that those who receive information may adjust their behavior, having consequences for the total contributions and quality provided, those that do not receive information may also update their beliefs across rounds and adjust their behavior accordingly. It is not clear, however, how this process is going to be, and whether it will amplify the effects of Hypothesis 3 or, on the other hand, change the direction of our predictions. This will be further investigated in our analysis.

\subsection{Implementation}

A fundamental feature of our experiment is the information provided to targeted individuals. So, in this section we describe the implementation of the multi-threshold public good game described in Section 3.1 but now with the information regimes introduced in Section 4.1. To implement targeting, a subset of subjects in each group in our information treatments is informed about the average of previous round contributions of their actual group members before making their own contribution decisions. Given our strangers matching protocol, the average contribution of their actual group members is computed using the individual contributions that each member of the current group made in the previous round. ${ }^{19}$ In addition to the explicit information provision in our information treatments, all subjects in all treatments are informed about the quality level reached once contribution choices have been made. However, subjects are never informed about the actual average contribution of their group members in the current round. ${ }^{20}$

Besides the No Info treatment already introduced in Section 3.3, we conducted additional sessions for the information treatments. Four sequences of our treatments were assembled, each of which involves two distinct information regimes. Table 3 shows how the rounds correspond to each information regime for the four sequences over all 46 rounds. Note that the regimes where information is provided are paired with the No Info regime in Sequences B, C, and D; whereas the No Info regime is paired with the Random Info

\footnotetext{
${ }^{19}$ Note that this "everybody else is doing it" notion is rooted in the perception of unfairness, and it occurs only when a subject perceives their peers' effort level or free riding. The different treatments in our experiment do nothing more than control some of the subjects' perceptions, arguably before he/she develops the "everybody else is doing it" notion. Within each treatment, if a subject is going to be exposed to peers' effort level, the question is what information should the subject receive. Specifically, we chose to create information from the previous round to reveal to the subjects. So, in effect, we treat every round in our experiment as a single period game with some subjects, depending on the treatment, receiving the information about their peers' free-riding behavior.

${ }^{20}$ Here we want to provide additional rationale for the strangers matching protocol we use given the additional informationaverage group contribution - provided to a subset of subjects in the information treatments. In a partners matching, the information provided would be the average group contribution in the previous round. As such, those not receiving information could still infer it when they are informed about the quality reached at the end of the round. To overcome this problem, a possible solution would be not to inform subjects about the average quality level at the end of the game. We did not believe it would be a reasonable solution as it might imply too much random play by subjects not informed about the outcome of the game.
} 
regime in Sequence A. We withheld information in the middle (rounds 17-31) in Sequences B-D to study the long-run "sustained" effects of information. Also, the sequences were designed such that subjects would not be "polluted" by other information regimes in the middle rounds before information is again reintroduced. Otherwise, we cannot safely tease apart the changes in outcomes to simply the same treatment in the third block of rounds.

Table 3: Experimental Treatments

\begin{tabular}{cccc}
\hline Sequence & Rounds 1-16 & Rounds 17-31 & Rounds 32-46 \\
\hline A & No Info & Random Info & No Info \\
B & Random Info & No Info & Random Info \\
C & Target Below & No Info & Target Below \\
D & Target Above & No Info & Target Above \\
\hline
\end{tabular}

The implementation of the sequences was identical to that discussed in Section 3.3, so the same setup applies here. However, there is an exception—beginning with Round 2-where information about average contributions to the public good is provided to a subset of subjects depending on the treatment. The provision of information follows beliefs to prevent the information from being a focal point in influencing beliefs. Also, the first round in each session has subjects receiving no information feedback (because we have no prior actions at that point).

In the information provision rounds (i.e., after Round 1), particular subjects in the targeted treatments that contribute below or above the average contribution receive information. We use the same algorithm for determining the number of participants that will receive information in the Random Info treatment. We do so in order to provide the same quantity of information to the subjects each period because this technique allows for direct comparison of results between the information treatments. More specifically, we count the number of subjects that would have been targeted, and then randomly select the same number of subjects for the randomly given information.

At Round 17, when the information treatments changed, subjects were explicitly notified and the supplemental instructions were distributed and read aloud by the experimenter. The subjects did not ex ante know if or when the treatment would change. When the treatments changed again in Round 32, subjects were again notified (but not whether/when they receive information).

For every round in the experiment, we followed the procedure described previously. Individual earnings from all 46 rounds were recorded by the computer and by subjects on their own information sheets. Toward 
the end of the experiment, three of the rounds were randomly chosen for payment. We did so to minimize wealth effects. Subjects' earnings in experimental tokens were then converted to US dollars at the rate of 20 tokens per dollar.

As before, the experiment was conducted at the Vernon Smith Experimental Economics Laboratory (VSEEL) at Purdue University in the spring of 2010, and the summer of 2012. Subjects were recruited by email using the laboratory's on-line recruitment system, and subject participation was limited to a single session. The computerized experimental environment was implemented using z-Tree (Fischbacher, 2007). Subjects were randomly assigned to individual computers and communication was not allowed during the session. Copies of the experiment instructions were provided to each subject and were read aloud by the experiment administrator. A copy of the instructions used to conduct the experiment is available in Appendix A, and a copy of the supplemental instructions is available in Appendix B. Completion of control questions was required to ensure each subject understood the experimental procedures prior to starting the actual experiment. Any subject that failed to answer the control questions after three attempts was personally assisted by the experiment administrator.

A total of 115 subjects participated in our experiments with each treatment comprised of 1 or 2 sessions (No Info: 20 subjects; Random Info: 25 subjects; Target Below: 40 subjects; Target Above: 30 subjects). The experiment lasted on average 1 hour and subjects were compensated between $\$ 8.25$ and $\$ 17.50$, with the average subject earning $\$ 12.94$. All subjects were paid in cash privately and individually at the conclusion of the experiment after completing a short demographic questionnaire.

\section{Experimental Results}

This section presents the analyses of our experimental data. Our main interest is to compare the information strategies and, for minimizing learning effects, we conduct a between-subjects analysis using the first block of rounds. Our analysis starts in Section 5.1 by describing aggregate behavior and comparing the effects between the different information regimes. After the aggregate results, Section 5.2 reports comparisons of contribution efficiency and also contribution waste. Then, in Section 5.3 we investigate the impact of receiving information within each information regime. The results presented in Sections 5.1, 5.2, and 5.3 represent the core of our experiment and tests of our hypotheses. Following that, we consider the withinsubjects analysis in Section 5.4, using all of the rounds, which allows us to investigate our secondary interest in understanding the effects of introducing, eliminating, and re-introducing information in our treatments. 
A summary of the main results are provided in Table 4 .

Table 4: Summary of Main Results

\begin{tabular}{|c|c|c|}
\hline Section & Objective & Main Results \\
\hline 5.1 & Comparisons of overall contribution performance & $\begin{array}{l}\text { Highest contributions in Target Above. Hypothesis } 3 \text { is } \\
\text { rejected. }\end{array}$ \\
\hline \multirow[t]{2}{*}{5.2} & $\begin{array}{l}\text { Comparisons of contribution efficiency and contribution } \\
\text { waste }\end{array}$ & $\begin{array}{l}\text { Cannot distinguish between No Info and Random Info. } \\
\text { Targeted treatments have higher efficiency and lower } \\
\text { waste compared to No Info. Hypothesis } 1 \text { is supported } \\
\text { for targeted treatments. }\end{array}$ \\
\hline & Comparisons of the average coefficient of variation & $\begin{array}{l}\text { Cannot distinguish between No Info and Random Info. } \\
\text { Hypothesis } 2 \text { is rejected. }\end{array}$ \\
\hline \multirow[t]{4}{*}{5.3} & Effect of receiving information & $\begin{array}{l}\text { Subjects adjust their contributions in response to infor- } \\
\text { mation. }\end{array}$ \\
\hline & $\begin{array}{l}\text { Comparison of targeted treatments, with or without in- } \\
\text { formation }\end{array}$ & $\begin{array}{l}\text { Indirect effect of information is significant for Target } \\
\text { Above. }\end{array}$ \\
\hline & Comparison of Above- and Below-Average Contributors & $\begin{array}{l}\text { Significant increase in contributions for below-average } \\
\text { contributors in Target Above. }\end{array}$ \\
\hline & Comparison of Early and Late Rounds for Random Info & $\begin{array}{l}\text { Possible explanation: mis-coordination of contributions } \\
\text { in Random Info in later rounds compared to No Info. }\end{array}$ \\
\hline 5.4 & Evaluate sustained effects of information & $\begin{array}{l}\text { Contributions do not significantly decline in Target } \\
\text { Above over time. }\end{array}$ \\
\hline
\end{tabular}

\subsection{Aggregate Comparisons Between Regimes}

We begin our analysis by presenting a graphical representation of the average contribution per round by treatment for Rounds 1-16 in Figure 4(a). To test for random assignment of subjects to treatments, we use the contribution behavior from Round 1 as no information had been provided to any subjects at the point in the experiment. A Kruskal-Wallis test confirms that we cannot statistically distinguish contributions between any of the treatments $\left(\chi^{2}=0.81, p\right.$-value $\left.=0.85\right)$. Additional comparisons between above-average contributors and between below-average contributors show that heterogeneous subjects are not assigned to treatments in a biased fashion. Kruskal-Wallis tests confirm that we cannot statistically distinguish aboveaverage contributors $\left(\chi^{2}=2.122, p=0.55\right)$ or below-average contributors $\left(\chi^{2}=1.753, p=0.63\right)$ between any of the treatments in Round 1. Based on these observations, we attribute that any changes in the outcomes are due to the provision of information in the treatments. Observe from the figure that Target Above and Target Below appear to have higher levels of contributions in comparison with Random Info and No Info. Similar patterns emerge for the average quality also (see Figure 4(b)).

Table 5 provides an aggregate representation of the average contribution and the average quality level for the four treatments for rounds 2-16-Round 1 is not included in these computations because we did 


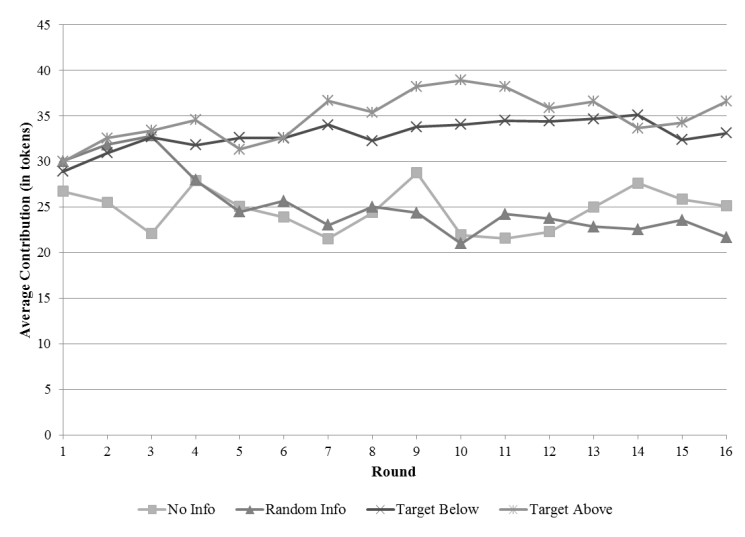

(a) Average Contribution per Round

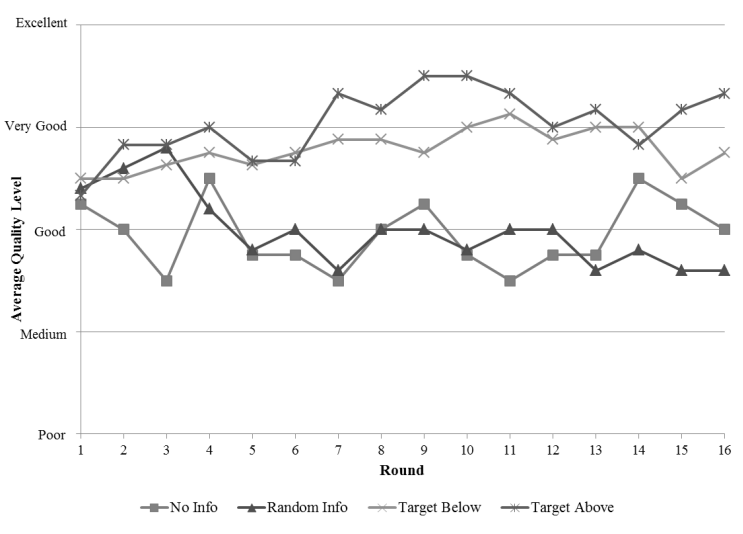

(b) Average Quality Level per Round

Figure 4: Per Round Measures

not provide any information in that round. As with Figure 4(a), the average contributions in No Info and Random Info are similar. Each of these treatments have a lower average contribution than Target Below and Target Above. The same pattern holds for the average quality levels as well.

Table 5: Average Contribution and Quality by Treatment in Rounds 2-16

\begin{tabular}{lccc}
\hline Treatment & Observations & Average Contribution & Average Quality \\
\hline No Info & 300 & $24.58(16.00)$ & $2.92(0.76)$ \\
Random Info & 375 & $24.99(15.19)$ & $2.96(0.83)$ \\
Target Below & 600 & $33.26(12.72)$ & $3.80(0.71)$ \\
Target Above & 450 & $35.26(13.68)$ & $4.09(0.76)$ \\
\hline
\end{tabular}

Standard deviations in parentheses.

Average quality obtained by treating the quality levels as discrete numbers.

We reinforce the findings from the aggregate results by estimating two random effects GLS models using panel data. We control for subject attributes such as age and gender, as well as use robust standard errors (clustered on each individual). The dependent variable in each regression is the individual contribution to the public good and we use No Info as the baseline treatment. Model I provides the effects of the treatment dummy variables that assign a value of one if the subject is in a particular treatment. ${ }^{21}$ Model II controls for

\footnotetext{
${ }^{21}$ Although non-parametric (two-tailed Mann-Whitney rank-sum) tests assume independence of observations, results are consistent with the parametric tests shown by Table 6 and do not show a statistically significant difference in both the average contribution and average quality provided in No Info and Random Info. Again, consistent with the regression, all of the other possible pairwise treatment comparisons for both the average contribution and average quality provided show a statistically significant difference at $p<0.001$. Treatment comparisons of the average contribution (quality) are: No Info vs. Random Info, $z=-0.44, p=0.66$ $(z=-0.92, p=0.36)$; No Info vs. Target Below, $z=-7.80, p<0.001(z=-14.92, p<0.001)$; No Info vs. Target Above, $z=-9.39, p<0.001$ ( $z=-16.62, p<0.001$ ); Random Info vs. Target Below, $z=-8.41, p<0.001(z=-14.96$, $p<0.001)$; Random Info vs. Target Above, $z=-10.03, p<0.001(z=-16.91, p<0.001)$; Target Below vs. Target Above, $z=-3.61, p<0.001(z=-6.14, p<0.001)$.
} 
beliefs about others' contributions. ${ }^{22,23}$ Based on the coefficient estimates from Table 6, the main results are consistent across the models and are as follows: No Info and Random Info are not statistically different, and No Info as well as Random Info lead to significantly lower contributions-in the statistical sense- than the targeted treatments.

Table 6: Random Effects GLS Regression on Contributions in Rounds 2-16: Baseline is No Info

\begin{tabular}{lcc}
\hline DV: Contributions & Model I & Model II \\
\hline Random Info & $0.22(3.32)$ & $1.70(2.86)$ \\
Target Below & $8.40^{* * *}(3.06)$ & $5.92^{* *}(2.78)$ \\
Target Above & $10.89^{* * *}(3.05)$ & $7.09^{* *}(2.79)$ \\
Beliefs & & $0.56^{* * *}(0.06)$ \\
Age & $0.37(0.47)$ & $0.33(0.34)$ \\
Gender & $-1.13(1.99)$ & $-1.50(1.61)$ \\
Constant & $16.90^{*}(10.16)$ & $1.80(7.25)$ \\
Observations & 1725 & 1725 \\
Subjects & 115 & 115 \\
$R^{2}$ & 0.10 & 0.34 \\
Wald $X^{2}$ & $26.78^{* * *}$ & $142.61 * * *$ \\
\hline
\end{tabular}

Regressions include subject-specific random effects.

Robust standard errors in parentheses.

*** $p<0.01, * * p<0.05, * p<0.10$

The result that Target Above generates a higher average contribution and quality than the other treatments indicates that the implication of Hypothesis 3 (and hence the hypothesis itself) does not hold. ${ }^{24}$ However, it does not directly imply that individuals do not have inequity aversion preferences (which we analyze later). Next we test Hypotheses 1 and 2 by analyzing group level measures of efficiency and coordination waste.

\subsection{Group Efficiency and Coordination Waste}

Recall from Section 3.4 that efficiency is defined as the surplus achieved relative to the maximum feasible surplus, and waste is the excess contributions above the threshold needed for the quality level achieved. Table 7 reports the average efficiency and average waste for each treatment by group. The average efficiency in No Info and Random Info are similar, and both of these treatments have a lower average efficiency than Target Below and Target Above. The same pattern holds for the average waste as well, with the exception that Target Below has only slightly less average waste than Random Info. Model I and Model II in Table

\footnotetext{
${ }^{22}$ Our use of beliefs as an independent variable is consistent with prior works in experimental economics when studying the factors that control for contributions in public goods (see e.g., Fischbacher and Gächter, 2010; Gächter and Renner, 2010).

${ }^{23} \mathrm{We}$ also created a model that used the average group contribution for those subjects that received period information in place of their stated beliefs. Results are qualitatively consistent with those presented in Table 6 .

${ }^{24}$ See also Table 1 in Appendix $\mathrm{C}$ for regressions using Target Below as the baseline treatment.
} 
8 estimate two random effects GLS models using panel data with robust standard errors clustered on each group. Both models use treatment dummy variables that assign a value of one if the subject is in a particular treatment. The dependent variable in Model I is group efficiency, whereas the dependent variable in Model II is group waste. No Info is used as the baseline treatment for both regressions. Results from the regressions comparing efficiency and waste show that we cannot reject the null hypothesis that the average efficiency and the average waste are the same across treatments. Efficiency is significantly higher for both Target Below and Target Above in comparison to No Info, and waste is significantly lower for Target Above in comparison to No Info. From these results, we find only partial support for Hypothesis $1 .^{25}$

Table 7: Efficiency, Coordination Waste, and Dispersion of Group Contributions in Rounds 2-16

\begin{tabular}{lcccc}
\hline Treatment & Obs. & Avg. Efficiency & Avg. Waste & Avg. Coef. of Variation \\
\hline No Info & 60 & $0.25(0.21)$ & $27.07(14.36)$ & $0.68(0.25)$ \\
Random Info & 75 & $0.26(0.22)$ & $26.96(14.61)$ & $0.62(0.31)$ \\
Target Below & 120 & $0.50(0.25)$ & $26.29(15.00)$ & $0.37(0.21)$ \\
Target Above & 90 & $0.61(0.29)$ & $21.87(15.49)$ & $0.38(0.25)$ \\
\multicolumn{4}{l}{ Standard deviations in parentheses. }
\end{tabular}

Table 8: Random Effects GLS Regression on Efficiency and Waste in Rounds 2-16: Baseline is No Info

\begin{tabular}{lccc}
\hline DV: & Model I: Efficiency & Model II: Waste & Model III: Coef. of Variation \\
\hline Random Info & $0.02(0.04)$ & $-0.11(1.60)$ & $-0.06(0.08)$ \\
Target Below & $0.25^{* * *}(0.05)$ & $-0.78(1.16)$ & $-0.31^{* * *}(0.08)$ \\
Target Above & $0.36^{* * *}(0.05)$ & $-5.20^{* *}(2.02)$ & $-0.30^{* * *}(0.10)$ \\
Constant & $0.24 * * *(0.03)$ & $27.07 * * *(0.65)$ & $0.68 * * *(0.07)$ \\
Observations & 345 & 345 & 345 \\
Groups & 23 & 23 & 23 \\
$R^{2}$ & 0.26 & 0.02 & 0.22 \\
Wald $X^{2}$ & $86.14 * * *$ & $6.77^{*}$ & $31.34^{* * *}$ \\
\hline
\end{tabular}

Regressions include group-level random effects.

Robust standard errors in parentheses.

$* * * p<0.01, * * p<0.05, * p<0.10$

Given that we hypothesized that random provision of information assists subjects in contributing more symmetrically, we next study how the variation of the contributions differ across the treatments. Because

\footnotetext{
${ }^{25}$ Although non-parametric tests may violate the assumption of independence of observations, results are consistent with the parametric tests for efficiency and waste. Mann-Whitney rank-sum tests to compare the efficiencies of treatments: No Info vs. Random Info, $z=-0.38, p=0.70 ;$ No Info vs. Target Below, $z=-5.47, p<0.001 ;$ No Info vs. Target Above, $z=-6.50$, $p<0.001 ;$ Random Info vs. Target Below, $z=-5.64, p<0.001$; Random Info vs. Target Above, $z=-6.77, p<0.001$; Target Below vs. Target Above, $z=-2.60, p=0.01$. Mann-Whitney rank-sum test for the treatment comparison of coordination waste by group are: No Info vs. Random Info, $z=0.11, p=0.91$; No Info vs. Target Below, $z=0.25, p=0.81$; No Info vs. Target Above, $z=2.05, p=0.04 ;$ Random Info vs. Target Below, $z=0.28, p=0.78 ;$ Random Info vs. Target Above, $z=2.14$, $p=0.03$; Target Below vs. Target Above, $z=2.03, p=0.04$.
} 
the means vary across the treatments, direct comparisons of variance may not be meaningful. So, here, we compare the average coefficient of variation, which measures the dispersion of contributions among group members. In addition to efficiency and waste, Table 7 also shows the average coefficient of variation of contributions across groups under each treatment. It provides evidence that the dispersion is much lower for the information treatments in comparison to No Info, with the exception of Random Info. Model III in Table 8 from our panel data regression reinforces the previous finding. The results indicate there is a statistically significant difference between all comparisons of No Info and the other treatments, with the exception of No Info versus Random Info. The information provided to subjects in targeted treatments serves to reduce dispersion, thereby improving the symmetry of the contributions in Target Below and Target Above. So, we reject Hypothesis 2 because the dispersion of contributions in Random Info is not significantly different than that for No Info. Next we consider the effects of information on contribution behavior.

\subsection{Effects of Receiving Information}

In Sections 5.1 and 5.2 we provided significant evidence of different levels of contributions and coordination in the information regimes. So, in this section, we investigate why outcomes are different by analyzing the effects of receiving information within each information regime. ${ }^{26}$ Recall, that in our information treatments only a subset of subjects receive information in each round. Subjects in Random Info are randomly selected to receive information, and subjects in Target Below (Target Above) recieve information if the subject contributed below (above) the average group contribution.

To determine the effect of information on contribution behavior, we create a new dependent variable to measure the change of contributions from last round to the current round $\left(\Delta\right.$ Contribution $_{i t}=$ Contribution $_{i t}-$ Contribution $\left._{i, t-1}\right)$. We conduct panel data regressions considering the effects of information and present the results in Table 9. The results are broken down by treatment. Note, we exclude Random Info from this regression because subjects that are above or below the group average respond simultaneously to information, thus offsetting the effects of information on contributions. Additional rationale for subject behavior in Random Info is explained later in this section. The table shows that, for Target Below, if subjects received information they increased their contributions; and the opposite is true for Target Above. These results appear to indicate that subjects are behaving consistent with inequity aversion. That is, if

\footnotetext{
${ }^{26}$ Our experimental design allows us to make what can be seen as a treatment-in-treatment analysis, where the "first treatment" concerns the different information regimes and the "second treatment" concerns whether a subject received information in a specific information regime.
} 
the subject knows they are below (above) the average for the group, they adjust their contribution level by increasing (decreasing) towards the average.

Table 9: Random Effects GLS Regression on Contribution in Rounds 2-16: Effect of Information

\begin{tabular}{lcc}
\hline DV: $\Delta$ Contribution $_{i t}$ & Target Below & Target Above \\
\hline Information & $6.28^{* * *}(1.20)$ & $-10.05 * * *(1.67)$ \\
Age & $0.20(0.13)$ & $0.23(0.24)$ \\
Gender & $-0.33(0.66)$ & $-0.19(1.07)$ \\
Constant & $-6.68^{* *}(3.12)$ & $1.33(5.01)$ \\
Observations & 600 & 450 \\
Subjects & 40 & 30 \\
$R^{2}$ & 0.11 & 0.15 \\
Wald $\chi^{2}$ & $27.74 * * *$ & $39.48^{* * *}$ \\
\hline
\end{tabular}

Regressions include subject-specific random effects.

Robust standard errors in parentheses.

*** $p<0.01, * * p<0.05, * p<0.10$

The previous result is shown visually by Figure 5, which plots the average of $\Delta$ Contribution $_{i t}$ for subjects with and without information in the information treatments. Note that (4) regions are labeled in the figure (Regions A-D), which we refer to in our upcoming comparisons. Regarding the figure, subjects that contributed above the group average now decrease their contribution in the next round (Region D: 4.01 token decrease by subjects receiving information in Target Above; Region B: -2.54 token decrease by subjects not receiving information in Target Below). We observe the opposite result for subjects that contributed below the group average (Region C: 5.96 token increase by subjects not receiving information in Target Above; Region A: 3.59 token increase by subjects receiving information in Target Below). The observed behavior in the targeted treatments is again consistent with inequity aversion concerns. Also, in Random Info, there is a decrease in contributions on average for those with information (-1.25 tokens), but there is practically no change in average contributions for those without information (0.005 token increase).

Next, we discuss two main results in order to setup comparisons between the targeted treatments: 1) contributions are higher in Target Above as compared to Target Below as evidenced in Section 5.1, and 2) receiving information seems to have a similar effect in both targeted treatments. Two observations are worth making. First, if the effect of information is the same, then the differences between the two information regimes seem to be driven by those not receiving information. Therefore, despite information appearing to have the same positive impact in both targeted treatments, it is possible that information may cause an indirect effect on contributions of those not receiving information, and that indirect effect may be higher in 


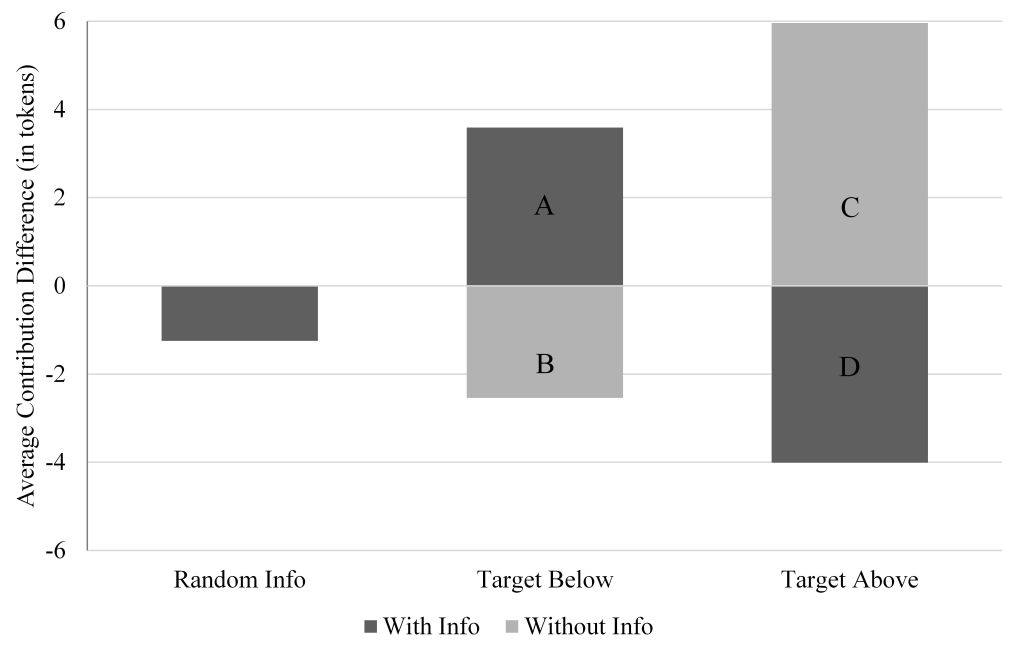

Figure 5: Average Difference in Contributions between Round $t$ and $t-1$ with and without Information for Targeted Treatments

the Target Above treatment.

So, in order to study the behavior of subjects who do not receive the information, we conduct two additional analyses. The first analysis corresponds to comparing across the bars in Figure 5, (i.e., Regions A vs. C; B vs. D), and the second analysis corresponds to comparing the impacts diagonally (i.e., Regions A vs. D; B vs. C).

Table 10: Random Effects GLS Regression on Contribution Difference With and Without Information in Rounds 2-16: Baseline is Target Below

\begin{tabular}{lcc}
\hline DV: $\Delta$ Contribution $_{i t}$ & With Info & Without Info \\
\hline Target Above & $1.14(1.53)$ & $2.85^{* *}(1.35)$ \\
Age & $-0.18(0.43)$ & $-0.24(0.16)$ \\
Gender & $-0.57(1.50)$ & $-0.11(1.22)$ \\
Constant & $8.44(9.44)$ & $8.74 * *(3.97)$ \\
Observations & 525 & 525 \\
Subjects & 64 & 64 \\
$R^{2}$ & 0.002 & 0.03 \\
Wald $\chi^{2}$ & 0.97 & $12.90^{* * *}$ \\
\hline Regressions include subject-specific random effects. \\
Robust standard errors in parentheses. \\
$* * * p<0.01, * * p<0.05, * p<0.10$
\end{tabular}

First, we compare the impact between treatments with and without information. For this analysis, we use $\Delta$ Contribution $_{i t}$ as defined earlier. However, note that the change in the contributions is opposite between Target Below and Target Above when information is provided. Therefore, we transform the contribution 
differences between treatments for subjects with information (i.e., (-1)(-4.01) tokens in target above versus 3.59 tokens in target below for Regions D vs. A) and those without information (i.e., 5.96 tokens in target above versus (-1)(-2.54) tokens in target below for Regions C vs. B). ${ }^{27}$ Results from the panel data analysis are shown in Table 10 and provide evidence that the difference in contributions from the prior round is significantly greater for those without information in Target Above compared to those without information in Target Below. Conversely, we cannot detect a measurable difference between treatments for those subjects that received information.

Table 11: Random Effects GLS Regression on Contribution Difference for Above- and Below-Average Contributors in Rounds 2-16: Baseline is Target Below

\begin{tabular}{lcc}
\hline & $\begin{array}{c}\text { Target Above With Info } \\
\boldsymbol{\&}\end{array}$ & $\begin{array}{c}\text { Target Above Without Info } \\
\boldsymbol{\&}\end{array}$ \\
DV: $\Delta$ Contribution $_{i t}$ & Target Below Without Info & Target Below With Info \\
\hline Target Above & $-1.21(1.68)$ & $2.56^{* *}(1.26)$ \\
Age & $0.38^{*}(0.20)$ & $0.05(0.34)$ \\
Gender & $-0.14(1.54)$ & $-0.91(1.19)$ \\
Constant & $-12.18^{* *}(4.75)$ & $3.44(7.68)$ \\
Observations & 573 & 477 \\
Subjects & 66 & 62 \\
$R^{2}$ & 0.01 & 0.01 \\
Wald $\chi^{2}$ & 4.40 & $7.94^{* *}$ \\
\hline Regressions include subject-specific random effects. \\
Robust standard errors in parentheses. \\
$* * * p<0.01, * * p<0.05, * p<0.10$
\end{tabular}

Second, we compare the differences in contributions between treatments receiving and not receiving information. If we compare subjects contributing above-average (Regions B vs. D), there is no difference between receiving and not receiving information (for this, we compare subjects' contributions in Target Above with information to subjects in Target Below without information. Results using panel data regressions that control for age and gender are presented in Table 11 and show that there is not a statistically significant difference with the difference being -1.47 tokens. However, there is a statistically significant difference in contributions amongst the subjects contributing below-average (Regions A vs. C) between receiving and not receiving information (5.96 versus 3.59 tokens). ${ }^{28}$

When both the analyses are taken together, it implies that any comparisons involving indirect informa-

\footnotetext{
${ }^{27}$ An alternative transformation such as absolute values of the contribution difference would shift the variance of the comparison, which is undesirable.

${ }^{28}$ A Mann-Whitney rank-sum test for Regions B vs. D also shows that there is not a statistically significant difference; $z=1.14$, $p=0.25$. There is a statistically significant difference for Regions A vs. C; $z=-2.01, p<0.05$ ).
} 
tion in the Target Above treatment are significant. It also indicates that the other three (Target Below direct and indirect information, and Target Above direct information) are not statistically different. Therefore, we reasonably conclude that indirect information in the Target Above treatment is the key factor leading to higher levels of contributions when compared with Target Below. So, those subjects contributing below the average in Target Above compensate for the reduction of contributions of those subjects that received information. A possible explanation follows that the subjects not receiving information are adjusting their contributions upwards because they may realize that others are contributing more than them (based on their quality provision). Therefore, they become more optimistic about maintaining the current quality level and/or reaching the next quality level.

As regards the effects of information in Random Info, we have already shown that subjects in Random Info have difficulty coordinating their contributions, based upon our coefficient of variation comparisons and rejection of Hypothesis 2 earlier. In Random Info, subjects receive information on both sides of the group average. So, any adjustments made by the subjects to the information are offset by adjustments made up or down by other subjects that also receive information. Further, if subjects react more strongly when they are above the group average compared to when they are below, information in Random Info may even have a negative effect. Therefore, in the next analysis we compare early and late round behavior in Random Info and No Info, to provide additional insight into why the ability to coordinate contribution levels is difficult in Random Info.

Table 12: Comparison of Early and Late Round Behavior for Random Info and No Info

\begin{tabular}{lccccc}
\hline & & \multicolumn{2}{c}{ Contribution } & & \\
\cline { 3 - 4 } Treatment & Rounds & Current Round & Last Round & Beliefs & Group Average \\
\hline Random Info & $2-16$ & 24.99 & 25.55 & 25.99 & 25.55 \\
No Info & $2-16$ & 24.58 & 24.69 & 28.77 & 24.69 \\
\hline Random Info & $2-6$ & 28.56 & 29.43 & 28.58 & 29.43 \\
Random Info & $12-16$ & 22.88 & 23.39 & 24.00 & 23.39 \\
No Info & $2-6$ & 24.90 & 25.47 & 29.11 & 25.47 \\
No Info & $12-16$ & 25.19 & 24.48 & 29.03 & 24.48 \\
\hline
\end{tabular}

Table 12 provides averages for Random Info and No Info. In Rounds 2 - 16 for Random Info, current contributions, last round contributions, and beliefs about other's contributions are all approximately the same. For the equivalent rounds in No Info, the averages are again similar to each other. Next, we segregate each of the treatments depending on whether they belong to the initial rounds $(2-6)$ or later rounds $(12-16)$. Focus primarily on the initial rounds. Between Random Info and No Info, the difference between treatments 
for both the current contributions and beliefs are not significantly different between Random Info and No Info for the initial rounds. However, a different result is observed in the later rounds for beliefs. From these observations, it appears that mis-coordination of contributions because of decreasing beliefs in Random Info during the later rounds is a concern in comparison to No Info, which we explain next.

The analysis shows that the dynamics of contribution behavior in Random Info are on average larger but not statistically dissimilar to No Info initially, suggesting that subjects may be trying to coordinate in both treatments. However, the eventual mis-coordination problems that arise in Random Info allow for the contributions to on average reduce but remain similar to No Info in later rounds, whereas beliefs are statistically different and higher for No Info. ${ }^{29}$ A possible explanation is as follows. When group contribution information is available, subjects tend to coordinate toward that information. When information is not available, subjects try to coordinate for the benefit of the group, or they decrease contributions less than compared to when they receive information. Initially, the contribution behavior in Random Info is higher than in No Info. However, in later rounds subjects are no longer optimistic about their ability to coordinate, as suggested by their beliefs. That is, a mis-coordination problem arises from the fact that subjects in Random Info are using information to coordinate contributions from above and below the group mean simultaneously. It follows that the simultaneous increase and decrease of contributions by subjects leads to contribution decay in Random Info because the contributions are missing the thresholds, and excess contributions are being wasted. That waste in turn leads pessimism towards meeting a higher threshold, and therefore to declining contributions. In the next section we consider the sustained effects of removing information.

\subsection{Effects of Withdrawing and Reintroducing Information}

Previous results showed that when information is targeted (either below or above the average), it generates higher contributions than providing it randomly. In the following, we investigate whether the provision of information has a lasting effect even if no information is provided in subsequent rounds. We also study whether re-introducing the information—after providing no information in the middle block of 15 roundsmaintains the consistency of the results. Thus, this section examines all of the rounds that subjects had played during the experiment. Here, we will refer to the sequences of treatments in the same manner as in Table 3, which is portrayed again in Table 13.

Table 13 presents the average contributions for each of the three blocks of rounds and the following

\footnotetext{
${ }^{29}$ See Table 2 in Appendix C.
} 
within-subjects analysis is performed using panel data regressions. ${ }^{30}$ For Sequence A, there is no significant difference between any of the blocks of rounds. Although the mean is slightly smaller in successive blocks, subjects generally appear to be maintaining the same level of contributions after moving to the random information treatment. The within-subjects comparison of the No Info and the Random Info treatments replicates the between-subjects comparison of the same two treatments that we presented earlier (see Table 5). In Sequence B, contributions degrade quite rapidly across the blocks. We observe statistically significant differences when comparing contribution averages in any two blocks. In Sequence C, there is not a statistically significant difference between the contribution averages of the first two blocks. When information is reintroduced, the average contribution is lower than that in the first and second blocks. Finally, in Sequence $\mathrm{D}$, the average contribution remains about the same (at a high level) across the blocks.

Table 13: Sustaining Coordination: Average Contributions

\begin{tabular}{clccc}
\hline & & \multicolumn{3}{c}{ Block of Rounds } \\
\cline { 3 - 5 } Sequence & Treatments & $\mathbf{2 - 1 6}$ & $\mathbf{1 7 - 3 1}$ & $\mathbf{3 2 - 4 6}$ \\
\hline A & No Info / Random Info / No Info & $24.58(16.00)$ & $23.67(16.23)$ & $21.21(15.91)$ \\
B & Random Info / No Info / Random Info & $24.99(15.19)$ & $19.42(15.52)$ & $11.81(14.35)$ \\
C & Target Below / No Info / Target Below & $33.26(12.72)$ & $32.38(12.91)$ & $30.77(14.66)$ \\
D & Target Above / No Info / Target Above & $35.26(13.68)$ & $36.25(13.67)$ & $34.96(14.84)$ \\
\hline
\end{tabular}

Standard deviations in parentheses.

Table 14: Random Effects GLS Regression on Contributions (Rounds 2-46): Baseline is Random Info

\begin{tabular}{lc}
\hline DV: Contributions & Coefficient \\
\hline Round & $-0.43^{* * *}(0.06)$ \\
Sequence C & $5.01 *(2.94)$ \\
Sequence D & $6.99 * *(3.05)$ \\
Round $*$ Sequence C & $0.35 * * *(0.07)$ \\
Round * Sequence D & $0.42 * * *(0.08)$ \\
Age & $0.33(0.48)$ \\
Gender & $-1.68(2.02)$ \\
Constant & $23.01 * *(10.57)$ \\
Observations & 4275 \\
Subjects & 95 \\
$R^{2}$ & 0.22 \\
Wald $X^{2}$ & $97.72 * * *$ \\
\hline Regression includes subject-specific random effects. \\
Robust standard errors in parentheses. \\
$* * * p<0.01, * * p<0.05 * p<0.10$
\end{tabular}

From Table 13, it seems that even though the contribution averages are not significantly different be-

\footnotetext{
${ }^{30}$ See Table 3 in Appendix C.
} 
tween Random Info versus No Info in the first block, provisioning information randomly at a later point in time (Round 17) slows the decay in contributions. To further explain these results, we follow the withinsubjects comparisons of blocks with a panel data regression. Table 14 shows the random effects GLS model assuming Random Info as the baseline. We find that the average contributions decline over time for the baseline (i.e., the coefficient for Round is statistically significant at $p<0.01$ ). In contrast, the contributions are not significantly declining with Round in Target Below or Target Above. Thus, Sequence C and Sequence D perform better than Random Info over time and after removing and reintroducing information, consistent with our earlier results for the targeted treatments.

\section{Conclusion}

In marketing, targeting aimed at nudging a participant to engage in a particular activity such as purchasing a product or service is universally well-known. Usually, the information provided seeks to explain the value of the product being marketed. In our context, we are motivated to study the "everybody else is doing it" attitude based on some contradicting observations in the piracy context. There are two related questions that we seek to answer. How does the information about others' free-riding behavior lead to an individual's free-riding? Can we nudge behavior based on providing targeted information to a subset of participants in the market? It turns out that the problem we began studying has broader contextual appeal. The insights we generate should be applicable to other information contexts where free-riding may occur such as crowdfunding, and open innovation.

Many of these contexts—-for example, piracy and crowdfunding—-have been shown to exhibit characteristics of a public good game. We, therefore, study the problem as a multi-threshold public good game. The game we present does not appear to have been studied in the literature and is also better suited to our context than other types of public good games studied before. We analyze the equilibrium for this game and also conduct the experiment when no information about others' contributions are provided. By implementing that experiment, we conclude that the multi-threshold game has coordination problems like other standard public good games.

Mechanisms—-such as the provision of information about other players' behavior-are sometimes studied in the prior literature to improve the ability of players to coordinate their actions in a game. In line with that and motivated by the "everybody else is doing it" attitude, we consider targeted information strategies. Specifically, we consider the following three treatments: 1) In Random Info, some participants are randomly 
selected for information; 2) In Target Below, participants whose contributions are below the average of the group, receive it; and 3) In Target Above, it is the opposite.

Results from the experiment are summarized as follows. We find that Random Info is not better than No Info from an average contribution standpoint, consistent with prior work (e.g., Sell and Wilson, 1991; Croson, 2001). A more surprising result is that targeted information strategies generate higher contributions, which initially appears to be in contrast with the predictions from strict inequity aversion or conformity (i.e., "everybody else is doing it" attitude) models. However, we do find that those subjects that receive information in the targeted treatments seem to act in accordance with inequity aversion. That is, subjects who receive information in Target Above decrease their contributions towards the group average, whereas those who receive information in Target Below act in the opposite manner. The Target Above treatment benefits from an indirect effect of information, where subjects that do not receive information still adjust their contributions upwards to maintain their current quality level and/or reach the next one. Compared with Target Below, the indirect effect of information is the key factor leading to higher levels of contributions in Target Above. Lastly, Random Info performs poorly because a mis-coordination problem arises due to subjects using information to attempt to coordinate contributions from above and below the group mean simultaneously. The simultaneous increase and decrease of contributions leads to contribution decay in Random Info because the contributions are missing the thresholds, and excess contributions are being wasted.

Overall, the conclusions from our paper provide evidence that it is possible to improve coordination in those settings where individuals may be targeted with information, ultimately leading to a more efficient (and socially desired) result. Although we do not find a significant difference in coordination in those cases where information is provided randomly versus not being provided at all, we do find differences between those treatments and the treatments that use targeted information. Therefore, it may be worthwhile to use targeted information strategies instead. Additionally, our research makes contributions to practice by asking questions of the status quo. For example, do the resources spent by industry groups on reporting the prevalence of piracy help or hurt their cause? Does the strategy of litigating individuals help in mitigating piracy? Also, what types and specificity of information should crowdfunding platforms use to improve the coordination of contributions towards projects by members of the platform?

In practice, policies related to our research have been or could be implemented in a variety of ways. For example, a software producer could utilize a support forum for their paying customers to provide information about piracy and thank them for doing the "right thing". Such a mechanism relates to Target Above treatment. 
Similarly, the Random Info treatment relates to the vendor putting out a press release or news announcement, where the producer does not know exactly the type of customer that is receiving the information. Incidents where artists such as Metallica berated fans from the stage about piracy (e.g., Jones, 2000) can be perceived to be equivalent to Random Info. We have also seen instances where artists such as Madonna poisoned the piracy networks with anti-piracy rants (instead of the actual song, Kaufman, 2003). These could be vehicles where the Targeted Below treatment can be executed.

Future research could extend our findings with those of Reuben and Riedl (2013), by considering the enforcement of contribution norms and targeted information strategies with heterogeneous populations. Researchers could also consider a field experiment to quantify the effects of targeted information provision. Furthermore, in practice, we may not be able to reach the entire group of people that are below or above an average level; however, if individuals in a group do not receive information, they may behave as if they do not receive information at all, so it might be worthwhile to study how interactions between the no information group and the targeted groups turn out. As with any research, ours has some limitations worth noting. Although we approximate the typical population of pirates by using university students, we cannot capture the entire range of potential pirates. Also, we used an abstract context for the implementation of the experiment to be sure that the observed effects in the experiment were only from our controlled manipulations, and therefore not affected by any potential framing effects.

\section{References}

2D Boy (2008). 90\%. http://2 dboy . com/2008/11/13/90/(accessed September 19, 2010).

Adomavicius, G., S. P. Curley, A. Gupta, and P. Sanyal (2012). Effect of information feedback on bidder behavior in continuous combinatorial auctions. Management Science 58(4), 811-830.

Adomavicius, G., S. P. Curley, A. Gupta, and P. Sanyal (2013). Impact of information feedback in continuous combinatorial auctions: An experimental study of economic performance. MIS Quarterly 37(1), 55-76.

Anderson, L. and S. Stafford (2009). An experimental study of the effect of announcements on public goods contributions. Working Paper.

Andreoni, J. (1990). Impure altruism and donations to public goods: A theory of warm-glow giving. The Economic Journal 100, 464-477.

Andreoni, J. and R. Croson (2008). Partners versus strangers: Random rematching in public goods experiments. In Handbook of Experimental Economics Results, Volume 1, Chapter 82, pp. 776-783. Elsevier B.V.

August, T. and T. I. Tunca (2008). Let the pirates patch? an economic analysis of software security patch restrictions. Information Systems Research 19(1), 48-70. 
Ayres, I., S. Raseman, and A. Shih (2012, August 20). Evidence from two large field experiments that peer comparison feedback can reduce residential energy usage. The Journal of Law, Economics, and Organization (Advance Access).

Bagnoli, M., S. Ben-David, and M. McKee (1992). Voluntary provision of public goods: The multiple unit case. Journal of Public Economics 47(1), 85-106.

Bapna, R., C. Dellarocas, and S. Rice (2010). Vertically differentiated simultaneous vickrey auctions: Theory and experimental evidence. Management Science 56(7), 1074-1092.

Bapna, R., J. Ramaprasad, G. Shmueli, and A. Umyarov (2012). One-way mirrors in online dating: A randomized field experiment. Workshop on Information Systems and Economics.

Berninghaus, S. K. and K.-M. Erhart (2001). Coordination and information: Recent experimental evidence. Economics Letters 73(3), 345-351.

Bhattacharjee, S., R. Gopal, J. R. Marsden, R. Sankaranarayanan, and R. Telang (2009). To theme or not to theme: Can theme strength be the music industry's "killer app"? Decision Support Systems 48(1), 141-149.

Bolton, G. E. and A. Ockenfels (2000). Erc: A theory of equity, reciprocity, and competition. American Economic Review 90(1), 166-193.

Brandimarte, L., A. Acquisti, and G. Loewenstein (2010). Misplaced confidences: Privacy and the control paradox. The $9^{\text {th }}$ Workshop on the Economics of Information Security.

Brandts, J. and D. Cooper (2006). A change would do you good: An experimental study on how to overcome coordination failure in organizations. American Economic Review 96(3), 669-693.

Burtch, G., A. Ghose, and S. Wattal (2013). An empirical examination of the antecedents and consequences of contribution patterns in crowd-funded markets. Information Systems Research 24(3), 499-519.

Burtch, G., A. Ghose, and S. Wattal (2014). The hidden cost of accommodating crowdfunder privacy preferences: A randomized field experiment. Management Science Forthcoming, 1-39.

Cason, T. N., K. N. Kannan, and R. Siebert (2011). An experimental study of information revelation policies in sequential auctions. Management Science 57(4), 667-688.

Cason, T. N. and F. U. Khan (1999). A laboratory study of voluntary public goods provision with imperfect monitoring and communication. Journal of Development Economics 58(2), 533-552.

Charness, G. (2004). Attribution and reciprocity in an experimental labor market. Journal of Labor Economics 22(3), 665-688.

Chellappa, R. K. and S. Shivendu (2005). Managing piracy: Pricing and sampling strategies for digital experience goods in vertically segmented markets. Information Systems Research 16(4), 400-417.

Chen, Y.-N. and I. Png (2003). Information goods pricing and copyright enforcement: Welfare analysis. Information Systems Research 14(1), 107-123.

Cho, W.-Y. and B.-H. Ahn (2010). Versioning of information goods under the threat of piracy. Information Economics and Policy 22(4), 332-340.

Cox, J. C. (2004). How to identify trust and reciprocity. Games and Economic Behavior 46(2), 260-281. 
Croson, R. (1996). Partners and strangers revisited. Economics Letters 53(1), 25-32.

Croson, R. (2001). Feedback in voluntary contribution mechanisms: An experiment in team production. Research in Experimental Economics 8, 85-97.

Croson, R., E. E. Fatás, and T. Neugebauer (2006). Excludability and contribution: A laboratory study in team production. Working paper, Wharton.

Croson, R. and M. Marks (1998). Identifiability of individual contributions in a threshold public goods experiment. Journal of Mathematical Psychology 42(2-3), 167-190.

Danaher, B., M. D. Smith, and R. Telang (2014). Piracy and copyright enforcement mechanisms. Innovation Policy and the Economy 14(1), 25-61.

Davis, D. and C. Holt (1993). Experimental Economics. Princeton University Press, Princeton, NJ.

Devetag, G. (2005). Precedent transfer in coordination games: An experiment. Economics Letters 89(2), 227232.

Devetag, G. and A. Ortmann (2007). When and why? a critical survey on coordination failure in the laboratory. Experimental Economics 10(3), 171-178.

Engelmann, D. and M. Strobel (2004). Inequality aversion, efficiency, and maximin preferences in simple distribution experiments. American Economic Review 94(4), 857-869.

Falk, A., E. Fehr, and U. Fischbacher (2000). On the nature of fair behavior. Economic Inquiry 4l(1), 20-26.

Falk, A. and J. J. Heckman (2009). Lab experiments are a major source of knowledge in the social sciences. Science 326, 535-538.

Fang, Y. and D. Neufeld (2009). Understanding sustained participation in open source software projects. Journal of Management Information Systems 25(4), 9-50.

Fehr, E. and S. Gächter (2000). Cooperation and punishment in public goods experiments. American Economic Review 90(4), 980-994.

Fehr, E. and K. M. Schmidt (1999). A theory of fairness, competition, and cooperation. The Quarterly Journal of Economics 114(3), 817-868.

Fischbacher, U. (2007). z-tree: Zurich toolbox for ready-made economic experiments. Experimental Economics 10(2), 171-178.

Fischbacher, U. and S. Gächter (2010). Social preferences, beliefs, and the dynamics of free riding in public goods experiments. The American Economic Review 100(1), 541-556.

Gächter, S. and E. Renner (2010). The effects of (incentivized) belief elicitation in public goods experiments. Experimental Economics 13(3), 364-377.

Gopal, R. and A. Gupta (2010). Trading higher software piracy for higher profits: The case of phantom piracy. Management Science 56(11), 1946-1962.

Hahn, J., J. Y. Moon, and C. Zhang (2008). Emergence of new project teams from open source software developer networks: Impact of prior collaboration ties. Information Systems Research 19(3), 369-391. 
Hashim, M. J., K. N. Kannan, S. Maximiano, and J. Rees Ulmer (2014). Digital piracy, teens, and the source of advice: An experimental study. Journal of Management Information Systems 31(2).

Herzenstein, M., U. M. Dholakia, and R. L. Andrews (2011). Strategic herding behavior in peer-to-peer loan auctions. Journal of Interactive Marketing 25(1), 27-36.

IFPI (2009). Digital music report 2009. http: / /www. ifpi.org/content/library/DMR2009-real. pdf (accessed January 3, 2012).

Isaac, R. M., D. Schmidtz, and J. M. Walker (1989). The assurance problem in a laboratory market. Public Choice 62(3), 217-236.

Isaac, R. M. and J. M. Walker (1988). Communication and free-riding behavior: The voluntary contribution mechanism. Economic Inquiry 26(4), 585-608.

Isaac, R. M., J. M. Walker, and A. W. Williams (1994). Group size and the voluntary provision of public goods. Journal of Public Economics 54, 1-36.

Jain, S. (2008). Digital piracy: A competitive analysis. Marketing Science 27(4), 610-626.

Johnson, J. P. (2002). Open source software: Private provision of a public good. Journal of Economics \& Management Strategy 11(4), 637-662.

Jones, C. (2000, April 13). Metallica rips napster. http://www.wired.com/politics/law/news/ $2000 / 04 / 35670$ (accessed September 9, 2011).

Kannan, K., M. Rahman, and M. Tawarmalani (2013). Implications of restricted patch distribution. Working Paper.

Kaufman, G. (2003, April 16). Madonna to pirates: 'what the $\mathrm{f}$ - do you think you're doing?' singer lashes out at file traders on p2p networks. MTV News http://www.mtv.com/ news/1471321/madonna-to-pirates-what-the-f-do-you-think-youre-doing/ (accessed December 21, 2015).

Khouja, M. and S. Park (2008). Optimal pricing of digital experience goods under piracy. Journal of Management Information Systems 24(3), 109-141.

Kosfeld, M., A. Okada, and A. Riedl (2009). Institution formation in public goods games. American Economic Review 99(4), 1335-55.

Lahiri, A. (2012). Revisiting the incentive to tolerate illegal distribution of software products. Decision Support Systems 53(2), 357-367.

Lahiri, A. and D. Dey (2013). Effects of piracy on quality of information goods. Management Science 59(1), 245-264.

Landsberger, H. A. (1958). Hawthorne revisited. Ithaca: Cornell University.

Levin, D. (2011). Rick Chazan on music piracy. Mess and Noise http://www.messandnoise.com/ articles/ 4172775 (accessed September 22, 2011).

Lin, M., N. R. Prabhala, and S. Viswanathan (2013). Judging borrowers by the company they keep: Friendship networks and information asymmetry in online peer-to-peer lending. Management Science 59(1), 17-35. 
Liu, Y., H. K. Cheng, Q. C. Tang, and E. Eryarsoy (2011). Optimal software pricing in the presence of piracy and word-of-mouth effect. Decision Support Systems 51(1), 99-107.

McCabe, K. A., M. L. Rigdon, and V. L. Smith (2003). Positive reciprocity and intentions in trust games. Journal of Economic Behavior \& Organization 52(2), 267-275.

Normann, H.-T. and H. A. Rau (2011). Step-level public goods: Experimental evidence. Working Paper.

Palfrey, T. R. and H. Rosenthal (1984). Participation and the provision of discrete public goods. Journal of Public Economics 24(2), 171-193.

Reuben, E. and A. Riedl (2013). Enforcement of contribution norms in public good games with heterogeneous populations. Games and Economic Behavior 77(1), 122-137.

Roberts, J. A., I.-H. Hann, and S. A. Slaughter (2006). Understanding the motivations, participation, and performers of open source software developers: A longitudinal study of the apache projects. Management Science 52(7), 984-999.

Sell, J. and R. K. Wilson (1991). Levels of information and contributions to public goods. Social Forces 70(1), 107-124.

Shang, J. and R. Croson (2009). A field experiment in charitable contribution: The impact of social information on the voluntary provision of public goods. The Economic Journal 119, 1422-1439.

Solomon, J., W. Ma, and R. Wash (2014). Don't wait! how timing affects coordination of crowdfunding donations. Working Paper.

Sundararajan, A. (2004). Managing digital piracy: Pricing and protection. Information Systems Research 15(3), 287-308.

Takahashi, S. (2010). Community enforcement when players observe partners' past play. Journal of Economic Theory 145(1), 42-62.

Telang, R. and J. Waldfogel (2014, August 6). Piracy and new product creation: A bollywood story. Working Paper.

Tsai, J. Y., S. Edelman, L. Cranor, and A. Acquisti (2011). The effect of online privacy information on purchasing behavior: An experimental study. Information Systems Research 22(2), 254-268.

Van Huyck, J. B., R. C. Battalio, and R. O. Beil (1990). Tacit coordination games, strategic uncertainty, and coordination failure. American Economic Review 80(1), 234-248.

Varian, H. R. (1998). Markets for information goods. Working Paper.

Wash, R. and J. Solomon (2014). Coordinating donors on crowdfunding websites. In Proceedings of the $17^{\text {th }}$ ACM Conference on Computer supported cooperative work \& social computing, pp. 38-48.

Weimann, J. (1994). Individual behavior in a free riding experiment. Journal of Public Economics 54(2), 185-200.

Zhang, J. and P. Liu (2012). Rational herding in microloan markets. Management Science 58(5), 892-912.

Zhang, X. and C. Wang (2012). Network positions and contributions to online public goods: The case of chinese wikipedia. Journal of Management Information Systems 29(2), 11-40. 


\section{Appendices: Information Feedback, Targeting, and Coordination: An Experimental Study}

\section{A Experiment Instructions}

This is an economic experiment about decision making under uncertainty. Listening carefully to these instructions will help you to earn a significant amount of money, which you will receive in cash privately at the end of the experiment. Your earnings in this experiment will depend on your performance in the individual rounds. Your final payout will be determined by three random draws done by the computer at the conclusion of the experiment. The three draws will correspond to three rounds during the experimental session. The total earnings over these three randomly selected rounds will be taken to calculate your final payout. All earnings in this experiment will be presented to you in tokens and converted to US dollars at the conclusion of the experiment. The conversion rate is: 20 tokens per 1 US dollar. The conversion rate is identical for everyone.

You are welcome to ask questions at any time by raising your hand. Please wait for an experimenter to come to your seat before asking your question. While the experiment is in progress, please do not speak or in any other way communicate with other participants. This is important to the validity of the study.

\section{Specific Guidelines:}

You will participate in 46 rounds in a group with four other participants. Participants are re-matched randomly at the beginning of each round to a new group of five participants. You will not know who is in your group. In each round you will receive an endowment of 50 tokens. The endowment is identical for everyone. You and every member in your group have to individually decide how much of this endowment to allocate to a group account. This allocation must be a whole number, between 0 and 50 tokens. All decisions are made simultaneously and without communication. No other group member will ever know how much you choose to allocate to the group account.

Your earnings in each round are determined by combining what is left of your endowment after the allocation, plus the consumption of a product. The earnings equation is presented below.

Your earnings $=$ endowment - your allocation + product quality value

The value from the product depends on the total group allocation. If the group allocation is between 0 and 49 , the quality of the product is Poor and the product quality value for you is 0 tokens. If the group allocation is between 50 and 99, the quality of the product is Medium and your product quality value is 18.5. If the group allocation is between 100 and 149, the quality of the product is Good and your product quality value is 45.5. If the group allocation is between 150 and 199, the quality of the product is Very Good and your product quality value is 81 . Lastly, if the group allocation is greater than 200 , the quality of the product is Excellent and your product quality value is 125 . These are summarized in the table below.

\section{Examples:}


1. If your combined group account for a round is 70 tokens, the quality of the product delivered to your group in that round is Medium. This will result in 18.5 tokens added as your Product Quality Value.

2. If your total payout for the three randomly chosen rounds is 232 tokens, you will earn $\$ 11.60$. In this case the experimenter will pay you a total of $\$ \mathbf{1 1 . 7 5}$ in cash at the conclusion of the experiment.

\begin{tabular}{ccc}
\hline $\begin{array}{c}\text { If your Total Group } \\
\text { Allocation is: }\end{array}$ & $\begin{array}{c}\text { Then your Product } \\
\text { Quality is: }\end{array}$ & $\begin{array}{c}\text { And your Product } \\
\text { Quality Value is: }\end{array}$ \\
\hline $0-49$ & Poor & 0 \\
$50-99$ & Medium & 18.5 \\
$100-149$ & Good & 45.5 \\
$150-199$ & Very Good & 81 \\
$200+$ & Excellent & 125 \\
\hline
\end{tabular}

Are there any questions? 


\section{B Supplemental Instructions}

Subjects receive the following supplemental instructions if their session begins with an information condition. These instructions will also be used after a restart if the subject's session began with a no feedback information treatment.

\section{IMPORTANT:}

Some participants in each group might receive information at the beginning of each round. If you receive information, you will see the average number of tokens the participants in your current group allocated to their prior group accounts in the previous round. The average number of tokens is presented as if you were in the same group last round.

\section{Examples:}

1. If every participant in your current group chose to allocate 10 tokens to the group account last round, the average allocation presented to you in the current round is 10 tokens.

2. If two participants in your current group chose to allocate 10 tokens to the group account last round, and three participants chose to allocate 20 tokens to the group account last round, the average token allocation presented to you in the current round is $16[=(10+10+20+20+20) / 5]$ tokens. 


\section{Supplemental Analyses}

Table 1: Random Effects GLS Regression on Contributions in Rounds 2-16: Baseline is Target Below

\begin{tabular}{lcc}
\hline DV: & Model I: Contributions & Model II: Quality \\
\hline No Info & $-8.40^{* * *}(3.06)$ & $-0.88^{* * *}(0.07)$ \\
Random Info & $-8.18^{* * *}(2.68)$ & $-0.83^{* * *}(0.08)$ \\
Target Above & $2.49(2.38)$ & $0.31^{* * *}(0.08)$ \\
Age & $0.37(0.47)$ & $0.01(0.01)$ \\
Gender & $-1.13(1.99)$ & $0.00(0.06)$ \\
Constant & $25.30^{* *}(10.04)$ & $3.48^{* * *}(0.32)$ \\
Observations & 1725 & 1725 \\
Subjects & 115 & 115 \\
$R^{2}$ & 0.10 & 0.30 \\
Wald $X^{2}$ & $26.78^{* * *}$ & $317.00^{* * *}$ \\
\hline Regressions include subject-specific random effects. \\
Robust standard errors in parentheses. \\
$* * * p<0.01, * * p<0.05, * p<0.10$
\end{tabular}

Table 2: Comparison of Early and Late Round Behavior using Random Effects GLS Regression on Contributions and Beliefs: Baseline is No Info

\begin{tabular}{lcccc}
\hline & \multicolumn{2}{c}{ Rounds 2-6 } & \multicolumn{2}{c}{ Rounds 12-16 } \\
DV: & Model I: Contributions & Model II: Beliefs & Model III: Contributions & Model IV: Beliefs \\
\hline Random Info & $3.85(3.37)$ & $-0.96(2.68)$ & $-2.66(4.40)$ & $-4.52^{*}(2.61)$ \\
Age & $0.46(0.51)$ & $0.13(0.34)$ & $-0.24(1.06)$ & $-0.38(0.85)$ \\
Gender & $0.84(3.50)$ & $-2.36(2.72)$ & $-1.78(4.73)$ & $2.83(2.84)$ \\
Constant & $14.89(11.24)$ & $26.73 * * *(7.93)$ & $30.55(22.99)$ & $36.81 * *(18.58)$ \\
Observations & 225 & 225 & 225 & 225 \\
Subjects & 45 & 45 & 45 & 45 \\
$R^{2}$ & 0.02 & 0.01 & 0.01 & 0.07 \\
Wald $X^{2}$ & 2.45 & 1.17 & 0.42 & 4.06 \\
\hline
\end{tabular}

Regressions include subject-specific random effects.

Robust standard errors in parentheses.

$* * * p<0.01, * * p<0.05, * p<0.10$ 
Table 3: Within-Subjects Comparison of Contributions using Random Effects GLS Regression (only significant comparisons are shown)

\begin{tabular}{lccccc}
\hline & Block 1 vs. 2 & Random Info & Block 1 vs. 3 & Block 2 vs. 3 & \multicolumn{2}{c}{ Target Below } \\
& $-5.57^{* * *}(1.56)$ & & & & \\
Block 1 vs. 3 & Block 2 vs. 3 \\
\hline Block 2 & & $-13.18^{* * *}(1.80)$ & $-7.61 * * *(1.56)$ & $-2.49 * *(1.16)$ & $-1.61 *(0.89)$ \\
Block 3 & $-0.25(0.84)$ & $-0.21(0.71)$ & $0.04(0.90)$ & $0.55(0.55)$ & $0.57(0.55)$ \\
Age & $-0.82(4.61)$ & $-0.48(4.12)$ & $0.59(4.46)$ & $-2.19(3.18)$ & $-2.20(3.39)$ \\
Gender & $70.19 *(18.10)$ & $29.48^{*}(15.33)$ & $18.63(19.08)$ & $21.23 *(11.79)$ & $19.91(11.92)$ \\
Constant & 750 & 750 & 750 & 1200 & 1200 \\
Observations & 25 & 25 & 25 & 40 & 40 \\
Subjects & 0.03 & 0.17 & 0.06 & 0.02 & 0.02 \\
$R^{2}$ & $12.83^{* * *}$ & $54.92^{* * *}$ & $24.07 * * *$ & 5.90 & 4.50 \\
Wald $X^{2}$ & & & & \\
\hline
\end{tabular}

Regressions include subject-specific random effects.

Robust standard errors in parentheses.

$* * * p<0.01, * * p<0.05, * p<0.10$ 